\title{
Selective Glycine Receptor $\alpha 2$ Subunit Control of Crossover Inhibition between the On and Off Retinal Pathways
}

\author{
Regina D. Nobles, ${ }^{1,2}$ Chi Zhang, ${ }^{1,3}$ Ulrike Müller, ${ }^{4,5}$ Heinrich Betz, ${ }^{4}$ and Maureen A. McCall ${ }^{1,2,3}$ \\ Departments of ${ }^{1}$ Ophthalmology and Visual Science, ${ }^{2}$ Psychological and Brain Sciences, and ${ }^{3}$ Anatomical Sciences and Neurobiology, University of \\ Louisville, Louisville, Kentucky 40202, ${ }^{4}$ Max Planck Institute for Brain Research, Department of Neurochemistry, 60528 Frankfurt am Main, Germany, and \\ ${ }^{5}$ Institute for Pharmacology and Molecular Biotechnology, Department of Bioinformatics and Functional Genomics, University of Heidelberg, 69120 \\ Heidelberg, Germany
}

In the retina, the receptive fields (RFs) of almost all ganglion cells (GCs) are comprised of an excitatory center and a suppressive surround. The RF center arises from local excitatory bipolar cell (BC) inputs and the surround from lateral inhibitory inputs. Selective antagonists have been used to define the roles of $\mathrm{GABA}_{\mathrm{A}}$ and $\mathrm{GABA}_{\mathrm{C}}$ receptor-mediated input in $\mathrm{RF}$ organization. In contrast, the role of glycine receptor (GlyR) subunit-specific inhibition is less clear because the only antagonist, strychnine, blocks all GlyR subunit combinations. We used mice lacking the GlyR $\alpha 2\left(\mathrm{Glra2}^{-/-}\right)$and GlyR $\alpha 3\left(\mathrm{Glra3}^{-/-}\right)$subunits, or both $\left(\mathrm{Glra2/3^{-/ }}\right)$, to explore their roles in GC RF organization. By comparing spontaneous and visually evoked responses of WT with Glra2 ${ }^{-/-}, \mathrm{Glra}^{-/-}$and $\mathrm{Glra2}^{-/ 3^{-/-}} \mathrm{ON}-$ and OFF-center GCs, we found that both GlyR $\alpha 2$ and GlyR $\alpha 3$ modulate local RF interactions. In the On pathway, both receptors enhance the excitatory center response; however, the underlying inhibitory mechanisms differ. GlyR $\alpha 2$ participates in crossover inhibition, whereas GlyR $\alpha 3$ mediates serial inhibition. In the Off pathway, GlyR $\alpha 2$ plays a similar role, again using crossover inhibition and enhancing excitatory responses within the RF center. Comparisons of single and double KOs indicate that GlyR $\alpha 2$ and GlyR $\alpha 3$ inhibition are independent and additive, consistent with the finding that they use different inhibitory circuitry. These findings are the first to define GlyR subunit-specific control of visual function and GlyR $\alpha 2$ subunit-specific control of crossover inhibition in the retina.

\section{Introduction}

The spatiotemporal interplay of excitatory and inhibitory inputs underlies both the receptive field (RF) organization and the sustained/transient nature of retinal ganglion cell (GC) responses. Together they provide the basis for their ability to extract information from the visual scene. Both GABA and glycine inhibition are used to modulate different aspects of GC responses and RF organization (Caldwell and Daw, 1978; Flores-Herr et al., 2001; O'Brien et al., 2003; Chen et al., 2010, 2011). Ionotropic GABA receptors (GABARs) and glycine receptors (GlyRs) are agonistgated chloride channels that provide the majority of this modulation. Both GABARs and GlyRs are composed of subunit combinations that create inputs with diverse kinetics, agonist affinities, and desensitization characteristics (Bormann, 2000; Webb and Lynch, 2007). The availability of selective antagonists have defined the roles of $\mathrm{GABA}_{\mathrm{A}} \mathrm{R}$ - and $\mathrm{GABA}_{\mathrm{C}} \mathrm{R}$-mediated in-

Received Oct. 23, 2011; revised Dec. 12, 2011; accepted Jan. 2, 2012.

Author contributions: R.D.N. and M.A.M. designed research; R.D.N., C.Z., and M.A.M. performed research; U.M. and H.B. contributed unpublished reagents/analytic tools; R.D.N., C.Z., and M.A.M. analyzed data; R.D.N., C.Z., H.B., and M.A.M. wrote the paper.

This work was supported by NIH Grant EY140701 (M.A.M.) and an unrestricted Research to Prevent Blindness grant to University of Louisville, Department of Ophthalmology and Visual Sciences. We thank Drs. Heinze Wässle, Stephen Mills, and Erika Eggers for critical reading of this manuscript.

The authors declare no competing financial interests.

Correspondence should be addressed to Maureen A. McCall, Department of Ophthalmology and Visual Sciences, 301 East Muhammad Ali Boulevard, University of Louisville, School of Medicine, Louisville, KY 40202. E-mail: mo.mccall@louisville.edu.

DOI:10.1523/JNEUROSCI.5341-11.2012

Copyright $\odot 2012$ the authors $\quad 0270-6474 / 12 / 323321-12 \$ 15.00 / 0$ hibition within the retinal circuit (for review, see Eggers and Lukasiewicz, 2011). GABARs are the receptor targets of amacrine cells (ACs), whose broad lateral processes ramify within one of the two sublamina (On or Off) of the inner plexiform layer (IPL) (MacNeil et al., 1999). GABAergic ACs target $\mathrm{GABA}_{\mathrm{A}} \mathrm{Rs}$ and $\mathrm{GABA}_{\mathrm{C}} \mathrm{Rs}$ in reciprocal inhibitory circuits to modulate RF center excitation (Eggers et al., 2007; Grimes et al., 2010). Similarly, they target $\mathrm{GABA}_{\mathrm{A}}$ Rs to modulate serial inhibition (Eggers and Lukasiewicz, 2010), to amplify and refine the RF surround (Cook and McReynolds, 1998; Roska et al., 2000; O’Brien et al., 2003).

GlyRs are the targets of glycinergic ACs whose narrow lateral processes ramify across the On and Off IPL sublaminae (MacNeil and Masland, 1998; Menger et al., 1998). GlyR inputs use crossover or feedforward inhibition to modulate GC RF center excitation (Wässle et al., 1986; Stone and Pinto, 1992; MacNeil and Masland, 1998; O’Brien et al., 2003; Chen et al., 2010; Russell and Werblin, 2010). The chloride channels formed by synaptic GlyRs are heteromeric and contain two $\alpha$ and three $\beta$ subunits (Grudzinska et al., 2005). GlyRs containing different $\alpha$ subunit isoforms ( $\alpha 1, \alpha 2, \alpha 3$, or $\alpha 4)$ display distinct IPL and neuronal subtype localization. Specifically, GlyR $\alpha 1$ is the predominant and perhaps only $\alpha$ subunit expressed by Off cone and rod bipolar cells (BCs) (Sassoè-Pognetto et al., 1994; Enz and Bormann, 1995; Cui et al., 2003; Ivanova et al., 2006). Although GlyR $\alpha 1$ modulates a significant inhibitory input to A-type murine GCs, GlyR $\alpha 2$, GlyR $\alpha 3$, and GlyR $\alpha 4$ also contribute to sIPSCs in these cells (Majumdar et al., 2007). Given the differences in localization, as well as current decay properties (for review, see Wässle et al., 2009) it 
is likely that GlyR subunits like $\mathrm{GABA}_{\mathrm{A}} \mathrm{R}$ and $\mathrm{GABA}_{C} \mathrm{Rs}$ participate in specific inhibitory mechanisms and circuits that control retinal function. Also like $\mathrm{GABA}_{\mathrm{A}} \mathrm{Rs}$, a lack of subunit-specific antagonists has prevented the definition of individual GlyR subunit-specific control of visual function.

Using a molecular genetic approach and GlyR $\alpha 2$ and $\alpha 3$ subunit knock-out (KO) mice, we defined subunit-specific inhibition in GC RF center responses. Our results define for the first time that (1) GlyR $\alpha 2$ and GlyR $\alpha 3$ inhibition enhance GC RF excitatory center responses, (2) GlyR $\alpha 2$ modulates crossover inhibition in both the On and Off pathways, and (3) GlyR $\alpha 2$ crossover and GlyR $\alpha 3$ serial inhibition are additive and independent in the On pathway.

\section{Materials and Methods}

Animals. We used male and female C57BL/6J wild-type (WT) mice (Jackson Laboratory) and two KO mouse lines that lacked GlyR $\alpha 2\left(\mathrm{Glra2}^{-/-}\right)$ (Young-Pearse et al., 2006) or GlyR $\alpha 3\left(\mathrm{Glra3}^{-1-}\right)$ (Harvey et al., 2004) subunit expression. The Glra2/3 ${ }^{-1-}$ double KO mice were generated by crossing and backcrossing the single $\mathrm{KO}$ lines. Genotypes were verified by PCR using primers and cycling conditions published previously (Harvey et al., 2004; Young-Pearse et al., 2006). All mice were maintained at the University of Louisville on a $12 \mathrm{~h}$ light/dark schedule. All experimental procedures were conducted in accordance with regulations described for the ethical care and treatment of animals in the Society for Neuroscience Policies on the Use of Animals in Neuroscience Research and in compliance with a protocol approved by the University of Louisville Institutional Animal Care and Use Committee.

Surgical preparation for electrophysiology recordings from optic nerve fibers. All surgical procedures were performed at light-adapted levels and have been published previously (Sagdullaev and McCall, 2005). Briefly, anesthesia was induced with an intraperitoneal injection of a Ringer's solution containing ketamine and xylazine. In WT mice, the induction dose was $127: 12 \mathrm{mg} / \mathrm{kg}$. In Glra2 ${ }^{-/-}$and $\mathrm{Glra}_{2} / 3^{-/-}$mice, an increase of $12.5 \%$ was required to induce a surgical plane of anesthesia. Anesthesia was maintained throughout the experiments with supplemental subcutaneous injections (50\% of initial concentration) administered every 45 min. The head was secured in a stereotaxic frame (David Kopf Instruments) with ear cups and a bite bar. Body temperature was maintained at $37^{\circ} \mathrm{C}$ with a feedback-controlled heating pad (TC-1000; CWE). Topical Mydfrin (phenylephrine hydrochloride ophthalmic solution, 2.5\%) and Mydriacyl (tropicamide ophthalmic solution, 1\%; Alcon Labs) were applied to dilate the pupils and paralyze accommodation. To prevent drying of the corneas, clear zero-powered lenses (Sagdullaev et al., 2004) moistened with artificial tears (Akwa Tears; Akorn) were placed over the eyes. A craniotomy was performed anterior to the Bregma suture and the overlying cortex was removed using suction to expose the optic nerve.

In vivo electrophysiology recordings from optic nerve fibers. Action potentials were recorded extracellularly from single optic nerve axons using sharpened tungsten electrodes (A-M Systems) with a final impedance between 30-100 M $\Omega$. A reference electrode was inserted subcutaneously. Action potentials from single GC axons were isolated, amplified (X3-Cell; FHC), digitized at $15 \mathrm{kHz}$ (Power1401; Cambridge Electronic Design), and stored for offline analysis. The isolated spikes were simultaneously displayed on an oscilloscope (60 MHz; Tektronix) and computer monitor (Spike2; Cambridge Electronic Design) and played over an audio monitor (AM7; Grass Instruments) to obtain direct feedback of the cell's response. Responses were analyzed offline using Spike2 software version 4.24 (Cambridge Electronic Design). Spikes were accumulated within a $50 \mathrm{~ms}$ bin width and displayed as poststimulus time histograms (PSTHs). Each average PSTH was smoothed by fitting it with a raised cosine function with a $50 \mathrm{~ms}$ smoothing interval to minimize alteration of the peak firing rate and maximize signal-to-noise ratio (Sagdullaev and McCall, 2005).

Quantitative characterization of GC visual response properties. The spatial extent and RF center sign of each GC was mapped using a handheld light source on a removable screen that covered a CRT display monitor
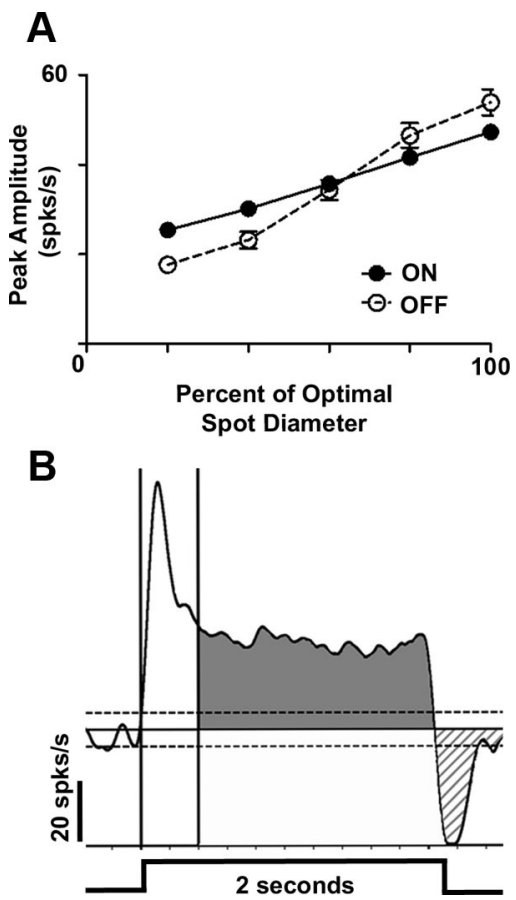

Figure 1. Representative area response function and PSTH response quantification during receptive field center stimulation. $\boldsymbol{A}$, Representative ARFs for WT ON (filled circles) and OFF (open circles) GCs. These ARFs plot the peak amplitude (defined as: peak - spontaneous firing rate) of the $\mathrm{GC}$ excitatory response as a function of the spot outer diameter up to the maximum response, which represents the spot diameter that matches the receptive field center size. This spot diameter is referred to as the optimal spot. $B$, A representative PSTH from a sustained ON GC to an optimal spot presented for a duration of $2 \mathrm{~s}$ (stimulus profile at bottom of PSTH). We defined the excitatory response when the firing rate crosses above $+3 \mathrm{SEM}$ of the mean spontaneous activity ( $\bar{x} S A$, top dotted line) until the response returns to the $\bar{x} S A$ (solid line). The vertical cursors define the transient response component $(0-0.4 \mathrm{~s})$. The shaded region represents the maintained response component $(0.4-2.0 \mathrm{~s})$. The hatched region (below $\bar{x} S A$ ) represents response suppression at stimulus offset when the spiking rate drops below -3 SEM $\bar{X} S A$ (bottom dotted line) until the response returns to the $\bar{X} S A$.

(Eizo E120 FlexScan FXC7). The screen was located $20-25 \mathrm{~cm}$ from the anterior nodal point of the eye. All stimulus and RF dimensions are corrected for monitor distance and are presented in the text in degrees of visual angle.

Receptive field center analysis. Computer generated stimuli (VisionWorks; Vision Research Graphics) were presented on a CRT monitor with a mean luminance of $20 \mathrm{~cd} / \mathrm{m}^{2}$. Stimulus luminance increased to $100 \mathrm{~cd} / \mathrm{m}^{2}$ for ON GCs and decreased to $3 \mathrm{~cd} / \mathrm{m}^{2}$ (67\% contrast) for OFF GCs. Stationary spots of increasing outer diameter (eight steps from 4.6 to $52.7^{\circ}$ ) were centered on the RF and presented in random order with randomly interleaved blank trials. Spots were presented for $2 \mathrm{~s}$ with a $5 \mathrm{~s}$ interstimulus interval. The mean spontaneous activity $(\bar{x} \mathrm{SA})$ was measured from "blank" trials, where the monitor luminance was $20 \mathrm{~cd} / \mathrm{m}^{2}$. \pm 3 SEM of the SA was used as a criterion to define thresholds for excitatory and suppressive responses.

Both peak and maintained firing rate were plotted as a function of spot diameter and formed an area response function (ARF) (Fig. 1A). We used the ascending limb of the ARF and its slope to evaluate spatial summation within the RF center, which results from the recruitment of excitatory $\mathrm{BC}$ input. The stimulus that elicited the maximum excitatory response defined the RF center diameter and is referred to as the optimal spot.

Excitatory response characteristics. We quantitatively characterized several aspects of the excitatory response from the average PSTH evoked by the optimal spot (Fig. 1B). Onset latency was defined as the time from stimulus onset until the time when the response rose to 3 SEM above $\bar{x}$ SA. Excitatory response duration was defined as the duration from response onset to offset, when the response decreased below $\bar{x} \mathrm{SA}$. The total excit- 


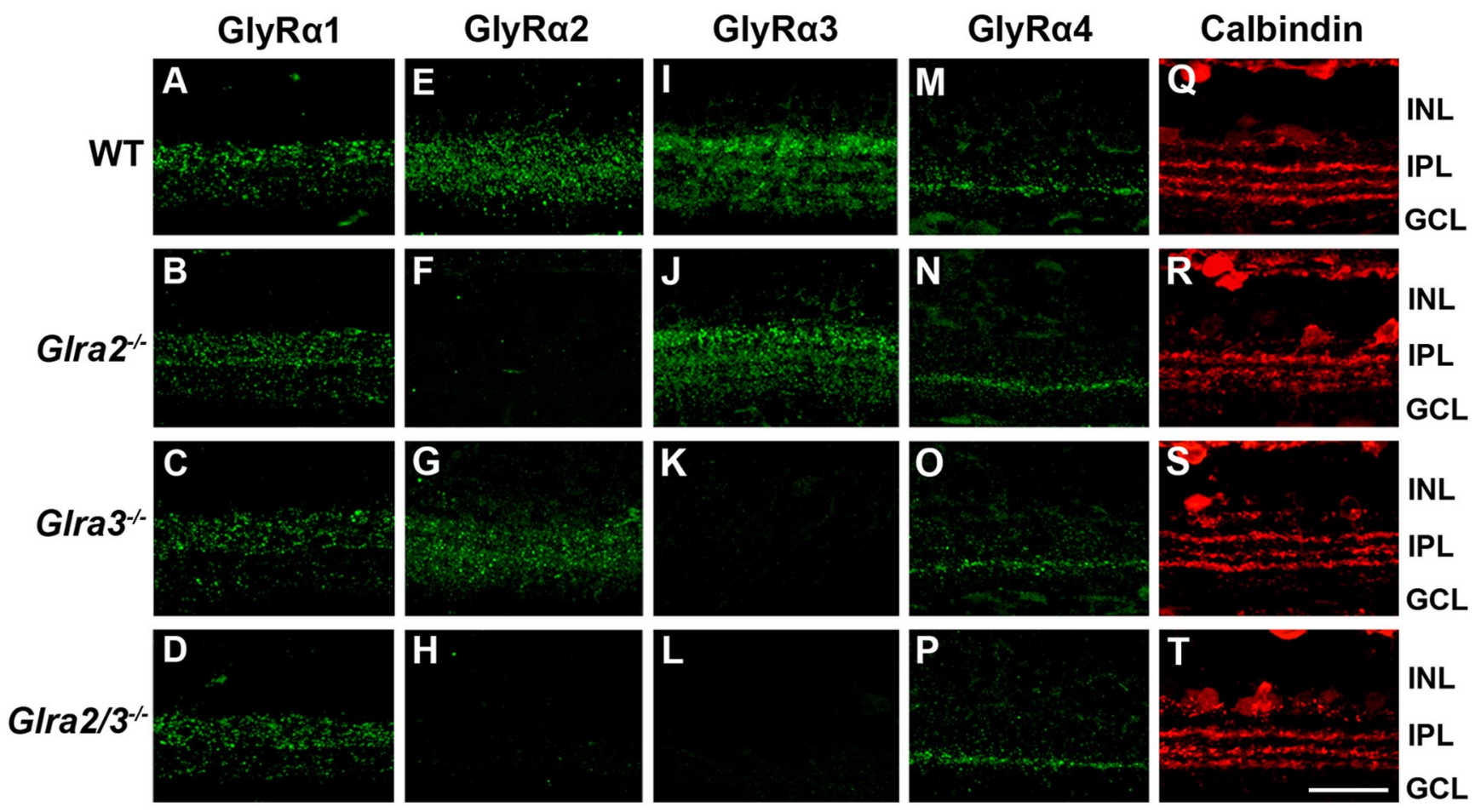

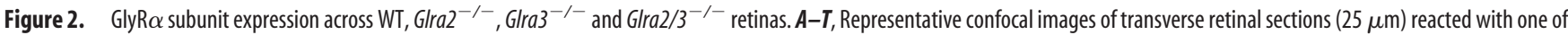
four GlyR $\alpha$ subunit-specific antibodies. INL, Inner nuclear layer; IPL, inner plexiform layer; GCL, ganglion cell layer. Scale bar, $20 \mu \mathrm{m}$. GlyR $\alpha 1$-immunoreactive puncta are prominent in the outer strata of the IPL across all genotypes $(\boldsymbol{A}-\boldsymbol{D})$. Representative confocal images of GlyR $\alpha 2$-immunoreactive puncta are diffuse across all IPL strata in WT and $G / r a 3^{-1-}$ retinas $(\boldsymbol{E}, \boldsymbol{G})$ and are absent in

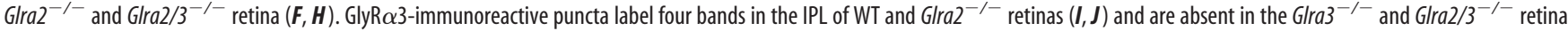
$(\boldsymbol{K}, \boldsymbol{L})$. GlyR $\alpha 4$-immunoreactive puncta are localized to a distinct band within the IPL in all genotypes $(\boldsymbol{M}-\boldsymbol{P})$. Retinas of all genotypes were reacted with an antibody to calbindin (red) and illustrate that IPL sublamination patterns are similar across genotype $(\mathbf{Q}-\mathbf{T})$.

atory response was defined as the area that remained 3 SEM above $\bar{x}$ SA. In WT GCs, the total excitatory response consisted of two components, a transient peak component (Fig. 1, white region), which then decayed to $37 \%$ of peak (or $1 / e$ ) within $0.4 \mathrm{~s}$ and a maintained component whose duration varied ( $>0.4$ to $\leq 2.0 \mathrm{~s}$; Fig. 1, gray region). Sustained cells were defined as those whose excitatory response remained 3 SEM above mean spontaneous level for the entire duration of stimulus presentation $(2 \mathrm{~s})$. Transient cells had excitatory responses that returned to the mean spontaneous level at $\leq 1.7 \mathrm{~s}$. We found no ganglion cells whose response durations were between $>1.7$ and $<2.0 \mathrm{~s}$.

Suppressive response characteristics. Using the same average PSTH, we quantified several aspects of response suppression that occurred at stimulus offset (Fig. $1 B$, hatched region). Onset latency of suppression was defined as the time from stimulus offset until the time when the response decreased below $\bar{x} S A$. The duration of response suppression was defined as the duration from onset latency of suppression until the response rose to $\bar{x} \mathrm{SA}$. The total magnitude of suppression was defined as the area when the response remained below $\bar{x} \mathrm{SA}$.

Immunohistochemistry. We examined the morphology of both singleand double $\mathrm{KO}$ retinas using fluorescence immunohistochemistry (Gregg et al., 2007). Retinas were removed from the eyecup, fixed for $15-20 \mathrm{~min}$ in $4 \%$ paraformaldehyde in $0.1 \mathrm{M}$ phosphate buffer $(\mathrm{PB}), \mathrm{pH}$ 7.4 , cryoprotected through a graded series of sucrose (Barthel and Raymond,1990), and embedded in OCT:20\% sucrose (2:1) (Sakura Finetek). Retinas from all four genotypes were stacked together in the same mold and frozen in OCT:20\% sucrose (2:1). Twenty-five micron transverse sections were cut through all four retinas on a CM 1850 cryostat (Leica) and mounted on microscope slides (Superfrost/Plus; Fischer Scientific). This ensured that each section was reacted under the same experimental protocol and was reacted with the exact same antibody concentration. Slides were brought to room temperature (RT), incubated in blocking solution ( $10 \%$ normal goat serum, $0.5 \%$ Triton X-100 in PBS) for 60 $\mathrm{min}$, and incubated with primary antibodies (diluted in blocking solu- tion) at RT overnight. A mouse monoclonal antibody to GlyR $\alpha 1$ (1:100; Synaptic Systems), goat polyclonal antibody to GlyR $\alpha 2$ and GlyR $\alpha 3$ (both1:50; Santa Cruz Biotechnology), and rabbit polyclonal antibody to GlyR $\alpha 4$ (1:100; Millipore Bioscience Research Reagents) labeled individual GlyR $\alpha$ subunits in WT and single and double KO retinas. A rabbit polyclonal antibody to calbindin (D-28k) (1:1000; Swant) labeled the sublamination pattern of the IPL. Sections were washed in PB and incubated in the secondary antibodies goat anti-mouse, donkey anti-goat, and goat anti-rabbit Alexa 488 in blocking solution for $90 \mathrm{~min}$. Sections were coverslipped with Immu-Mount (Thermo Scientific), and images were taken on an Olympus BX51WI confocal microscope equipped with a Hamamatsu CCD EM digital camera using a $40 \times$ PLAPO WLSM objective and FluoView version 5.0 software (Olympus).

Isolation of RNA and reverse transcription. Both retinas were removed from WT and $\mathrm{Glra3}^{-/-}$mice and pooled. Total RNA was extracted from each sample using TRI Reagent $(1.0 \mathrm{ml}$ per two retinas) and purified using RiboPure Kit (Ambion). The quantity and quality of the RNA was determined using a NanoDrop 2000 spectrophotometer (Thermo Fisher Scientific). First-strand cDNA synthesis was performed by a reaction of $350 \mathrm{ng}$ of total RNA from each sample using the SuperScript III Reverse Transcriptase (Invitrogen).

Real-time PCR. Real-time PCR was performed on a total of $20 \mu \mathrm{l}$ of cDNA in reaction mix in 96-well plates using 7900HT Fast Real-Time PCR System (Applied Biosystems). Each $20 \mu \mathrm{l}$ reaction mixture included $1 \mu \mathrm{lcDNA}, 2 \mu \mathrm{l}$ TaqMan Fast Universal PCR Master Mix, and $0.5 \mu \mathrm{l} 20 \times$ TaqMan assay containing either GlyR $\alpha 2$ or $18 \mathrm{~s}$ RNA primer sets (GlyR $\alpha 2$, Mm00806742_m1; 18s, Mm03928990_g1; Applied Biosystems). For each cDNA sample, three to four PCR replicates were performed using each primer set. The PCR parameters were: incubation at $50^{\circ} \mathrm{C}$ for $2 \mathrm{~min}$, denaturation at $95^{\circ} \mathrm{C}$ for $10 \mathrm{~min}$, and 40 cycles of $95^{\circ} \mathrm{C}$ for $15 \mathrm{~s}$ and $60^{\circ} \mathrm{C}$ for $1 \mathrm{~min} . \Delta C_{\mathrm{T}}$ of GlyR $\alpha 2$ in each cDNA sample was normalized to the rRNA $18 \mathrm{~s}$ as an endogenous control as follows: $\Delta C_{\mathrm{T}}=$ $C_{\mathrm{T}, \text { GlyR } \alpha 2}-C_{\mathrm{T}, 18 \mathrm{~s}}$. Here, $C_{\mathrm{T}}$ is the cycle threshold. The $\Delta C_{\mathrm{T}}$ ratio was 
ON

$\mathrm{Ai}$

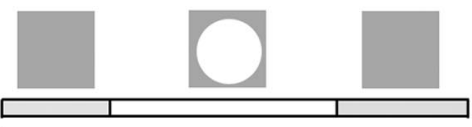

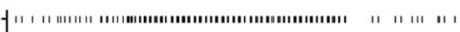

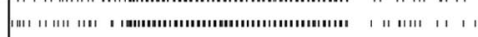

-

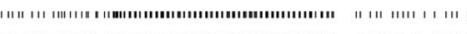

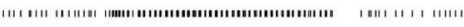
ห1

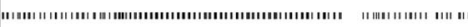

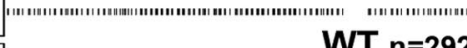

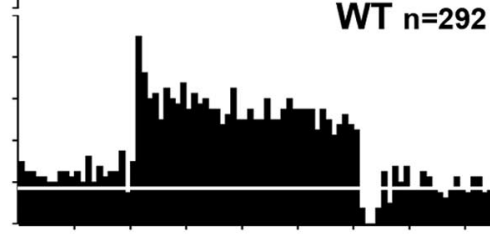

Aii

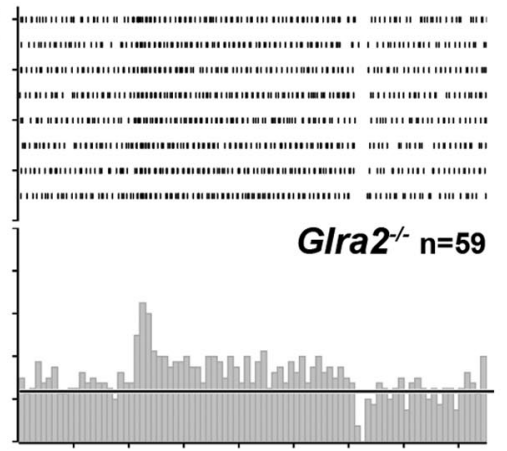

Aiii

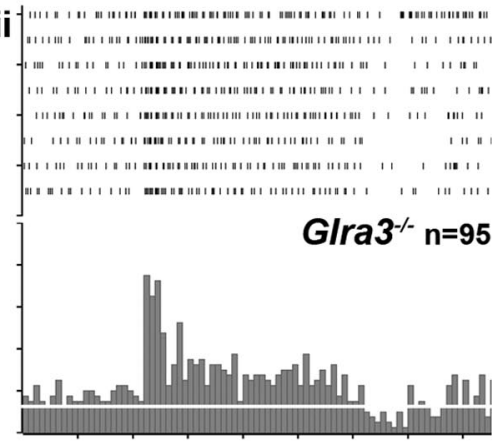

\section{Aiv}

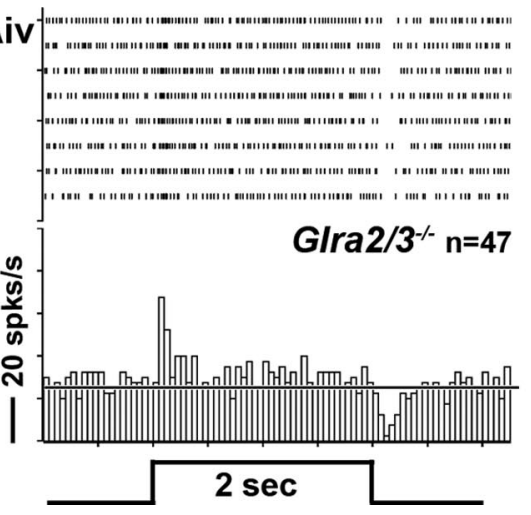

OFF sustained

$\mathrm{Bi}$

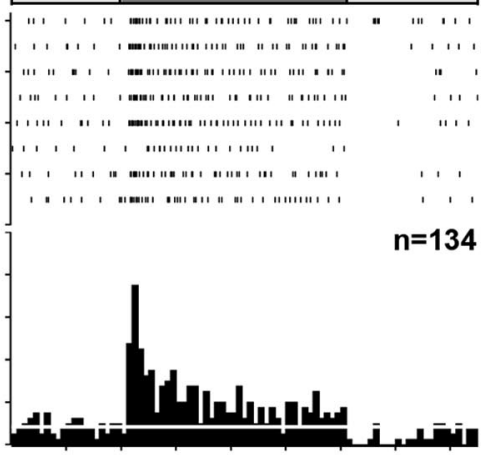

$\mathrm{Bii}$

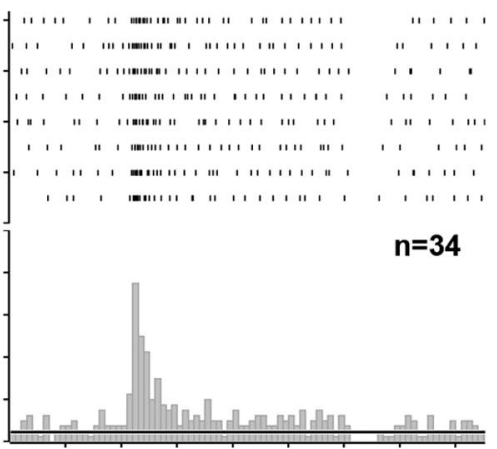

Biii

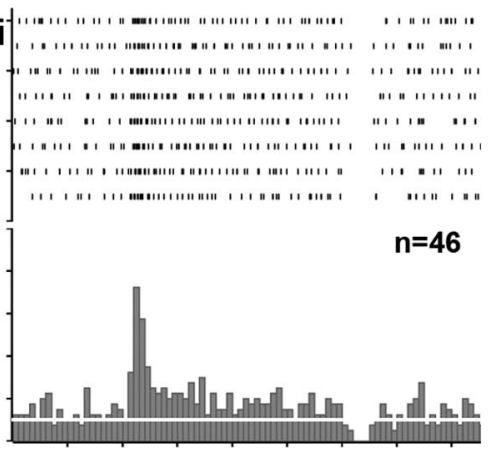

Biv
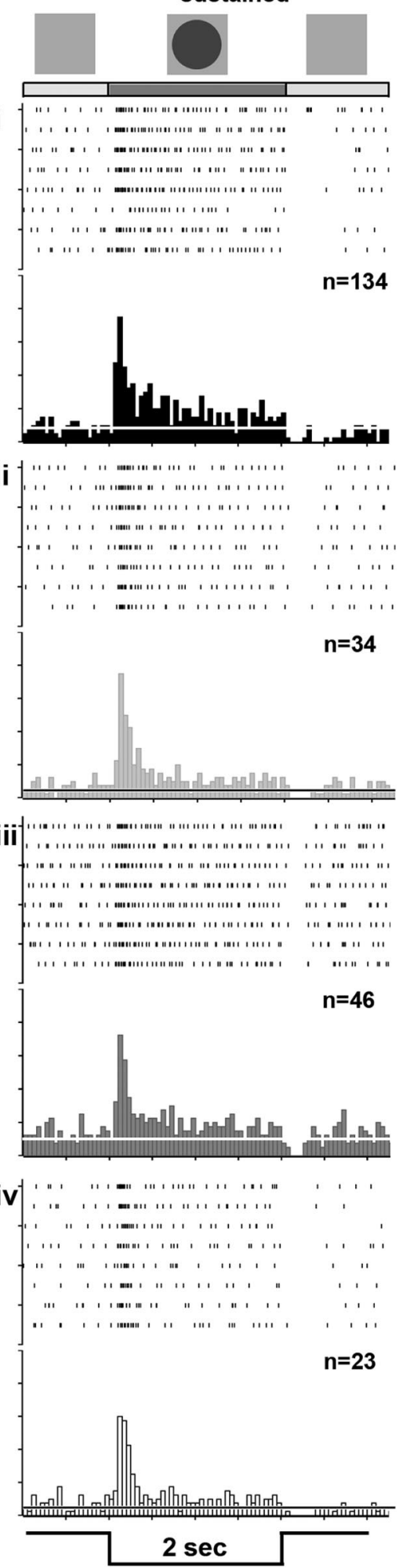

OFF transient

$\mathbf{C i}_{\mathbf{i}}$

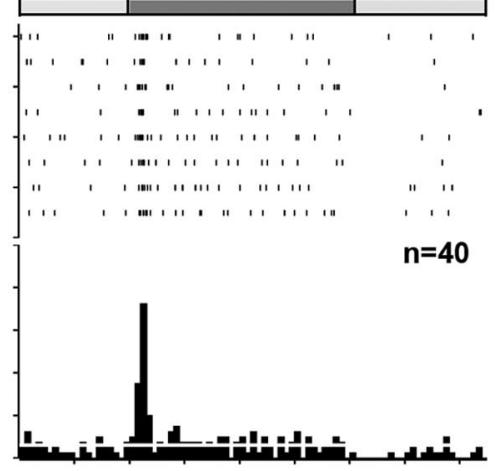

Cii

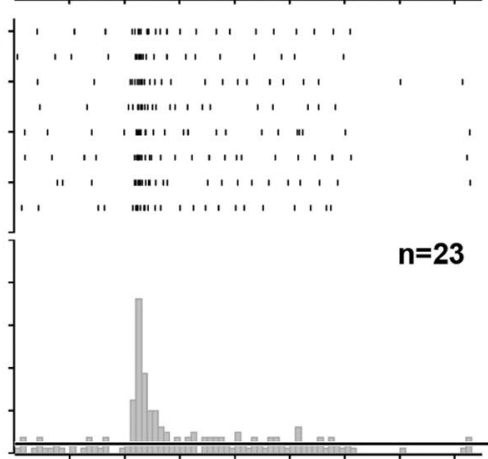

Ciii

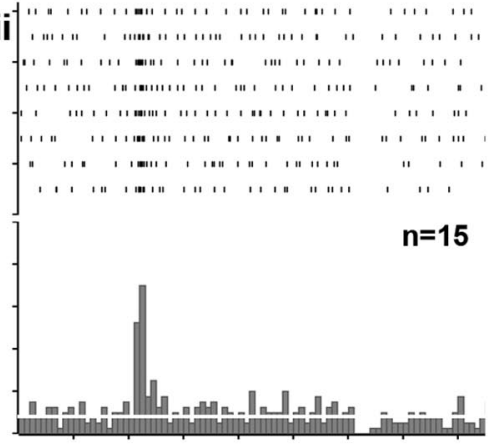

Civ

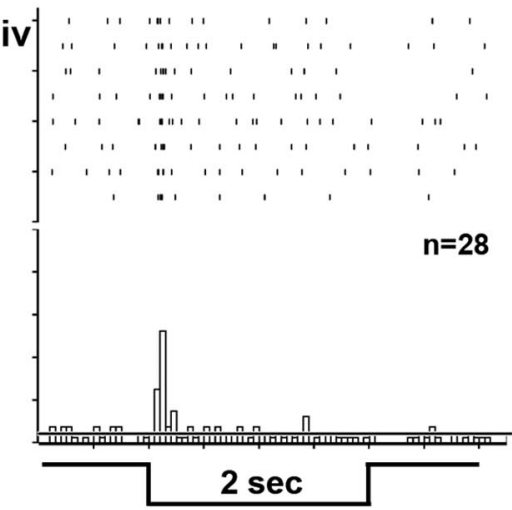

Figure 3. Receptive field center excitatory responses of $\mathrm{ON}, 0 \mathrm{FF}_{\text {sustained }}$ and $\mathrm{OFF}$ transient $\mathrm{GCS}$ across genotype. Average PSTHs for ON and OFF $\mathrm{GCs}$ evoked by their optimal spot are shown along with the eight individual responses in raster plots (above). The luminance profile of the stimulus ( 2 s duration) is shown at the bottom of each column. For $0 \mathrm{NGCs}$, a bright spot ( $100 \mathrm{~cd} / \mathrm{m}^{2}$ ) was presented, and for OFF GCs, a dark spot $\left(3 \mathrm{~cd} / \mathrm{m}^{2}\right)$ was presented on a screen whose average luminance was $20 \mathrm{~cd} / \mathrm{m}^{2}$. Scale bar, 20 spikes per second. Ai-Aiv, ON GCs of all genotypes increase spiking at the onset of a bright spot and their excitatory response remains above the $\bar{X} S A$ (solid line) for the entire duration of the stimulus. Bi-Civ, OFF GCs increase spiking at the onset of dark spot centered on their RFs and can be divided by the duration of their response compared to the stimulus. Bi-Biv, $0 \mathrm{FF}_{\text {sustained }} \mathrm{GCS}$ respond for the entire duration of the stimulus presentation. Ci-Civ, $0 \mathrm{FF}$ transient $\mathrm{GC}$ responses decline to the $\bar{x} \mathrm{SA}$ before stimulus offset $(\leq 1.70 \mathrm{~s})$. 

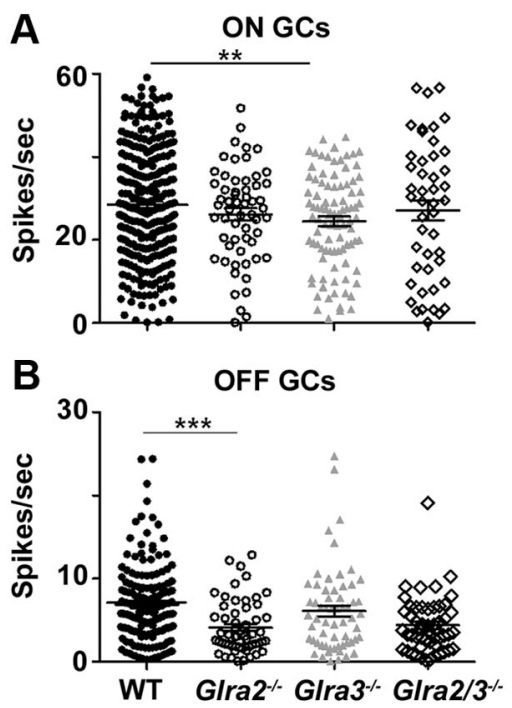

Figure 4. GlyR $\alpha 3$ expression selectively governs $\mathrm{ON}$ and $\mathrm{Gly} R \alpha 2$ expression governs $\mathrm{OFF} \mathrm{GC}$ spontaneous activity. $A, 0 N \mathrm{GCS}$. Spontaneous activity is significantly lower in $\mathrm{Glra}^{-/-} \mathrm{ONGCS}$ (gray triangles; $n=94$ ) compared to WT GCs (filled circles; $n=291$ ), but is similar between WT and $\mathrm{Glra2}^{-\prime-}$ ON GCS (open circles; $n=58$ ). The spontaneous activity levels do not differ between double KOs (open diamonds; $n=46$ ) and single KOs. B, OFF GCs. Spontaneous activity is significantly lower in GIra2 ${ }^{-1-}$ OFF GCS (open circles; $n=57$ ) compared to WT GCs (filled circles; $n=171$ ), but similar between WT and Glra ${ }^{-/-}$OFF GCs (gray triangles; $n=61$ ). The spontaneous activity levels in double KO OFF GCs (open diamonds; $n=47$ ) are similar to those in $\mathrm{Glra2}^{-/-}$OFF GCs and single K0s. ${ }^{* *} p<0.01 ;{ }^{* * *} p<0.001$.
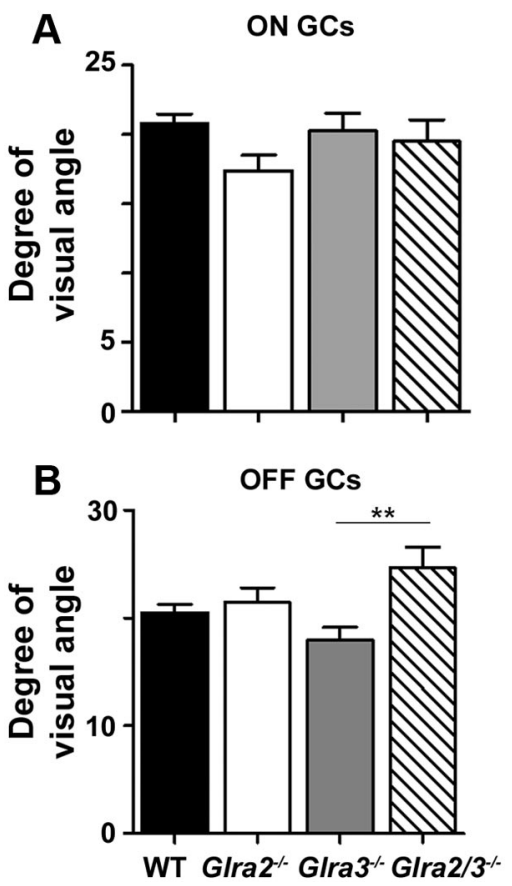

Figure 5. Receptive field center diameter is not affected by the absence of either GlyR $\alpha 2$ or GlyR $\alpha 3$ expression. A, ON GCs. The RF center diameters of both single KO ON GCS (Glra2 ${ }^{-/-}$, open bar, $n=59 ;$ Glra $^{-1-}$, gray bar, $n=95$ ) are similar to those of WT (black bar; $n=292$ ). The RF center diameters of double KO ON GCs (hatched bar; $n=47$ ) were similar to those of both single KO ON GCS. B, OFF GCS. The RF center diameters of both single KO OFF GCS (Glra2 ${ }^{-1-}$, open bar, $n=57 ;$ Glra3 $^{-1-}$, gray bar, $n=61$ ) are similar to those of WT (black bar; $n=174$ ). The RF center diameters of double KO OFF GCS (hatched bar; $n=51$ ) are similar to those of $\mathrm{GIra2}^{-/-}$OFF GCS, but are significantly larger than those of $\mathrm{Glra}^{-/-}$OFF GCS. ${ }^{* *} p<0.01$.
Table 1. Receptive field center diameter (degree of visual angle)

\begin{tabular}{|c|c|c|c|c|c|c|c|c|c|c|c|c|}
\hline \multirow[b]{2}{*}{$\mathrm{GC}$ class } & \multicolumn{3}{|l|}{ WT } & \multicolumn{3}{|c|}{$\mathrm{GIra2}^{-1-}$} & \multicolumn{3}{|c|}{$\mathrm{Glra3}^{-1-}$} & \multicolumn{3}{|c|}{ Glra2/3-1- } \\
\hline & $\bar{x}$ & $\pm S E$ & $n$ & $\bar{x}$ & $\pm S E$ & $n$ & $\bar{x}$ & $\pm S E$ & $n$ & $\bar{x}$ & $\pm \mathrm{SE}$ & $n$ \\
\hline ON & 20.8 & 0.7 & 292 & 17.4 & 1.1 & 59 & 20.3 & 1.2 & 95 & 19.5 & 1.6 & 47 \\
\hline OFF & 20.5 & 0.7 & 174 & 21.4 & 1.4 & 57 & 18.0 & 1.2 & 61 & 24.7 & 1.9 & 51 \\
\hline
\end{tabular}

The spot outer diameter that elicited the maximum spiking response for ON and OFF GCs is an estimate of the RF center diameter for each genotype. The mean RF diameter $(\bar{x}), \pm$ SE, and total number $(n)$ of cells are presented for each genotype. RF dimensions are corrected for monitor distance and are presented in degrees of visual angle.

obtained by dividing each $\Delta C_{\mathrm{T}}$ by the lowest $\Delta C_{\mathrm{T}}$ from the results. The $\Delta C_{\mathrm{T}}$ ratios of WT and $\mathrm{Glra3}^{-1-}$ mice were compared using a nonparametric $t$ test to examine differences in transcriptional level of GlyR $\alpha 2$.

Statistical analyses. Statistical analyses were performed using GraphPad Prism5 software version 5.04 for Windows. Distributions of each response component were tested for normality using the D'AgostinoPearson omnibus test. The distributions of our quantitative responses across GC types and mouse strains were not normal. As a consequence, wherever possible, we used nonparametric statistical analyses to test for differences. We compared each single KOs to WT and double KOs to each single $\mathrm{KO}$ using an unpaired $t$ test with Welch's correction ( $t$ test with Welch's). Because our sample size across spot diameter satisfies the central limit theorem, we used a two-way ANOVA to compare responses across spot diameter between genotypes. A linear regression analysis was used to compare changes in the slope of the RF center summation between genotypes. We used a $\chi^{2}$ analysis to determine any differences in the distributions of transient versus sustained OFF GCs between genotypes. Data in graphs are represented as mean \pm SEM.

\section{Results}

Normal inner retinal morphology is retained in the absence of GlyR $\alpha 2$, GlyR $\alpha 3$, or both receptor subunits

In the Glra3 ${ }^{-/-}$mice used in these studies, the absence of GlyR $\alpha 3$ retinal expression has been demonstrated previously (Harvey et al., 2004). In the Glra2 $2^{-/-}$mice used here, the absence of retinal expression was verified at the transcription level at P0 (Young-Pearse et al., 2006). We used immunostaining with subunit-specific antibodies to confirm the absence of GlyR $\alpha 2$ and GlyR $\alpha 3$ expression in adult Glra2 $^{-/-}$(Fig. 2, second row), Glra3 ${ }^{-/-}$(third row) and Glra2/3 $3^{-/-}$ (fourth row) retina sections compared to WT (Fig. 2, first row). Each single KO lacked the characteristic immunoreactivity of its deleted GlyR $\alpha$ subunit, and the double KOs exhibited neither GlyR $\alpha 2$ nor GlyR $\alpha 3$ staining. In both the single and the double $\mathrm{KO}$ retinas, the expression patterns of the GlyR $\alpha 1$ and GlyR $\alpha 4$ subunits did not differ from WT (Fig. 2, first and fourth columns). Because GlyR $\alpha$ subunits are expressed primarily in the IPL, we examined and compared the calbindin sublamination pattern in the IPL across WT and single and double KO retinas. Retinas from all four genotypes showed three clear calbindinimmunoreactive bands (Fig. 2, fifth column). Thus, the gross morphology of the inner retina appears intact regardless of the absence of GlyR $\alpha 2$, GlyR $\alpha 3$, or both subunit expression. The GlyR $\alpha 3$ antibody labels four discrete bands in the IPL (Haverkamp et al., 2003), and although moderate upregulation of GlyR $\alpha 2$ expression has been reported across the IPL in the absence of GlyR $\alpha 3$ (Heinze et al., 2007), this difference was not evident in our immunohistochemical analysis.

GlyR $\alpha 2$ transcription levels are not altered in $\mathrm{Glra3}^{-/-}$mice We used real time PCR to examine the expression of the Glra2 gene in WT retina and to determine whether it is upregulated in Glra3 ${ }^{-/-}$retina. Our results show that the level of GlyR $\alpha 2$ mRNA in WT $(n=9)$ and Glra3 ${ }^{-/-}(n=8)$ retinas $\left(\Delta C_{\mathrm{T}}\right.$ ratio $)$ was similar (WT, $1.029 \pm 0.01$; Glra3 $^{-/-}, 1.046 \pm 0.016 ; p=0.13$ ). The electrophysiological data presented below are consistent 

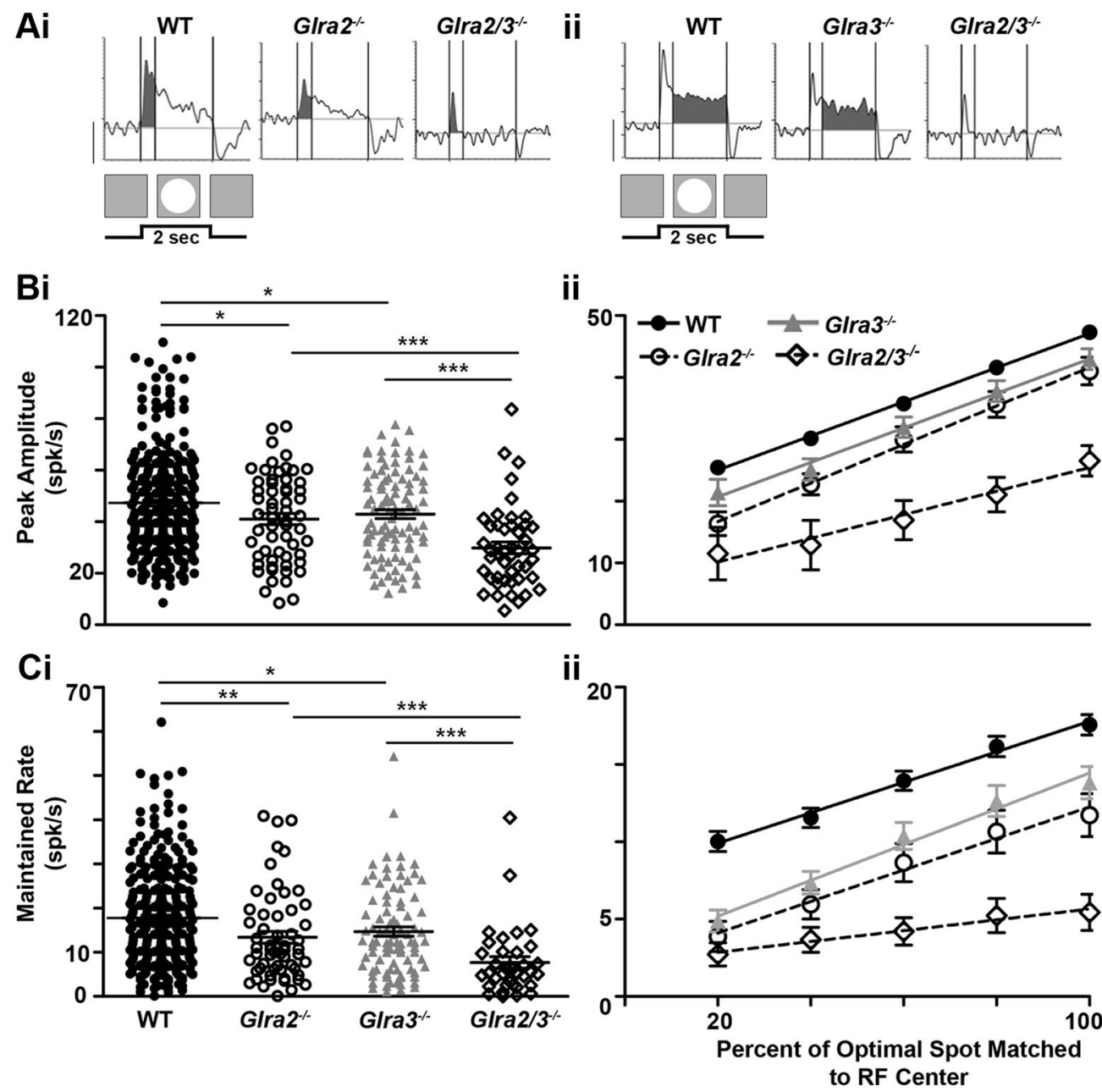

Figure 6. Both GlyR $\alpha 2$ - and GlyR $\alpha 3$-mediated inhibition enhances the receptive field center responses of ON GCs. Ai, Aii, Representative PSTHs of WT and single- and double K0 ON GC RF responses. Shaded regions represent the transient $(0-0.4 \mathrm{~s})$ response component (Ai) and the maintained response component $(0.4-2 \mathrm{~s}$; $A$ iii). The stimulus (a bright spot) and its duration profile are shown below the response. $\mathbf{B i}$, The scatter plot shows the distribution of the peak amplitude responses across genotype and illustrates the differences across genotype. The peak amplitudes of

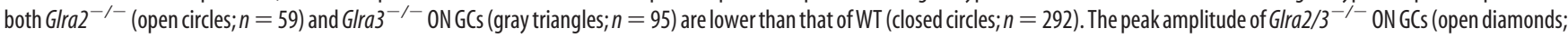
$n=47$ ) is lower than the peak amplitude for either single K0. Bii, The mean ARFs for each genotype. RF diameter is normalized so that the optimal diameter is plotted at $100 \%$. The slope represents the rate of change in peak amplitude during RF center summation and is similar between WT and single K0s ( $p=0.07$ ) but differs between single and double K0s ( $p<0.001$ ). All curves were analyzed using a linear regression and the goodness of fit for all genotypes; $R^{2}=0.99$. Ci, The scatter plot shows the distribution of maintained firing rates across genotype and the differences across

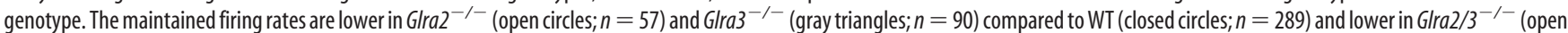
diamonds; $n=34$ ) compared to single KO ON GCS. (ii, The mean ARFs for each genotype. RF diameter is normalized so that the optimal diameter is plotted at $100 \%$. The slope represents the rate of change in maintained firing rate during RF center summation and is similar between WT and single KOs $(p=0.30)$ but differs between single and double KOs ( $p<0.0001)$. All curves were analyzed using a linear regression and the goodness of fit for all genotypes; $R^{2}=0.98$. Scale bar, 20 spikes per second. ${ }^{*} p<0.05 ;{ }^{* *} p<0.01 ;{ }^{* * *} p<0.001$.

with this result and suggest that there is no upregulation of GlyR $\alpha 2$ in the absence of GlyR $\alpha 3$.

GlyR subunit-specific inhibition enhances ON and OFF GC spontaneous activity but does not alter receptive field center size

Because little is known about the role of GlyR subunits in GC spontaneous or visually evoked responses, we examined and compared the responses of GCs using spot stimuli of varying diameter to probe their RF center response. We divided GCs into those with ON-center (ON) or OFF-center (OFF) RFs and their responses into excitatory and suppressive phases (Fig. $1 B$ ). Representative responses of each GC class recorded from all four genotypes are shown in the average PSTHs of Figure 3. Raster plots above each PSTH indicate their spiking responses to individual stimulus presentations. Among our 292 WT ON GCs, we found only 10 cells with transient responses. We divided the excitatory responses of OFF GCs into transient and maintained components relative to the presence of the stimulus. For each GC response characteristic, we compared WT ON or OFF GCs (Fig. $3 A i, B i, C i$ ) to GCs from each single KO (Fig. $3 A i i-C i i i)$. This allowed us to determine whether one or both subunits modulated the response. We used comparisons of GC responses between single (Fig. 3Aii-Ciii) and double KOs (Fig. $3 A i v-C i v$ ) to evaluate whether GlyR $\alpha 2$ and GlyR $\alpha 3$ inhibition was independent. Finally, we compared changes in excitatory and suppressive responses in an attempt to determine the synaptic circuit(s) that could account for the changes observed.

The SA of OFF GCs was significantly lower than ON GCs in WT as well as all knock-outs. The SA of $\mathrm{Glra3}^{-/-}$ON GCs was significantly lower than that of WT ON GCs (Fig. 4A; $24.5 \pm 1.2$ spikes/s, $n=94$ vs $28.5 \pm 0.8$ spikes/s, $n=291 ; p=0.005)$. The SA of $\mathrm{Glra2}^{-/-}$OFF GCs was significantly lower than that of WT 
OFF GCs (Fig. $4 B ; 4.1 \pm 0.4$ spikes/s, $n=57$ vs $6.8 \pm 0.3$ spikes/s, $n=174 ; p<0.001)$. The SA of Glra2/3 ${ }^{-1-}$ ON $(27.1 \pm 2.5$ spikes/s; $n=46)$ and OFF GCs (4.7 \pm 0.5 spikes/s; $n=47)$ was similar to that of single $\mathrm{KO}$ ON $\left(\mathrm{Glra2}^{-/-}, p=0.70\right.$; $\mathrm{Glra3}^{-/-}$, $p=0.30)$ and OFF GCs (Glra2 $\left.{ }^{-/-}, p=0.47 ; \mathrm{Glra3}^{-/-}, p=0.08\right)$ (Fig. $4 A, B$ ). Our results indicate that GlyR $\alpha 3$ and GlyR $\alpha 2$ subunits provide pathway-specific tonic inhibition. GlyR $\alpha 3$ acts within the On pathway, whereas GlyR $\alpha 2$ acts within the Off pathway. Each serves to enhance the tonic spontaneous firing rate of GCs in the WT retina. In light of this finding in the subsequent analyses, SA was subtracted from evoked GC responses in all genotypes.

The RF center diameters of Glra2 $2^{-/-}$and $\mathrm{Glra3}^{-/-} \mathrm{ON}$ (Fig. $5 A$ ) and OFF (Fig. 5B) GCs were similar to those of WT GCs (Table 1; ON, $p=0.11, p=0.51$; OFF, $p=0.56, p=0.08$, respectively). The RF diameters of $\mathrm{ON}$ single and double KO GCs also were similar ( $p=0.50$ and $p=0.95$, respectively). The mean RF diameters of double KO OFF GCs, while similar to Glra2 ${ }^{-/-}$ $(p=0.44)$, were significantly larger than Glra3 $^{-/-}(p=0.005)$. Our results are primarily consistent with previous studies that GlyR modulation does not contribute significantly to RF center spatial tuning (O'Brien et al., 2003; Manookin et al., 2008; van Wyk et al., 2009; Chen et al., 2010). The difference that we observe in the RF diameters of the double KO OFF GCs could be confounded with their generally low response amplitudes and more transient responses, which could lead to the need for larger spots to obtain responses significantly above SA.

\section{WT ON GCs receptive field center excitation is enhanced by both GlyR $\alpha 2$ and GlyR $\alpha 3$ inputs}

The onset of a stationary bright spot whose dimensions are within or match the RF center evokes an excitatory response in WT ON GCs. In the overwhelming majority of WT ON GCs in our sample (282 of 292) this excitatory response has an initial transient peak within $0.40 \mathrm{~s}$ of stimulus onset (Fig. 6 Ai, left) followed by a maintained excitation that is temporally matched to the stimulus $(2 \mathrm{~s})$ (Fig. 6Aii, left). The remaining 10 WT ON GCs had only a transient peak.

The transient peaks of both Glra2 ${ }^{-/-}(41.0 \pm 2.2$ spikes/s; $n=$ $59)$ and Glra3 ${ }^{-1-}$ ON GCs $(43.0 \pm 1.7$ spikes/s; $n=95)$ were significantly lower than those of WT ON GCs (Fig. 6Bi; $47.3 \pm$ 1.2 spikes $/ \mathrm{s} ; n=292 ; p=0.02$ and $p=0.04$, respectively). The maintained firing rates of both Glra2 $^{-/-}(13.4 \pm 1.3$ spikes/s; $n=$ 57) and Glra3 ${ }^{-1-}$ ON GCs $(14.7 \pm 1.0$ spikes/s; $n=90)$ also were significantly lower than those of WT ON GCs (Fig. 6Ci; $17.8 \pm$ 0.7 spikes $/ \mathrm{s} ; n=289 ; p=0.005$ and $p=0.01$, respectively). The difference in both peak and maintained firing rates are evident for both $\mathrm{Glra2}^{-/-}$and $\mathrm{Glra3}^{-/-}$GCs across spot diameters smaller than the RF center, however only the maintained rates are significantly lower (Fig. 6Cii; $p<0.01$ ). These results suggest that the input of both GlyR $\alpha 2$ and GlyR $\alpha 3$ subunits augments the total excitatory response in WT ON GCs.

The peak amplitude $(30.0 \pm 2.3$ spikes $/ \mathrm{s} ; n=47)$ and maintained firing rates $(7.0 \pm 1.0$ spikes/s; $n=34)$ of $\mathrm{Glra} 2 / 3^{-/-} \mathrm{ON}$ GCs were significantly lower than those of either single KO (Fig. $6 \mathrm{Bi}, \mathrm{Ci} ; p<0.001)$. This result indicates that GlyR $\alpha 2$ and GlyR $\alpha 3$ subunit inputs contribute separately to the RF center excitatory response of WT ON GCs. They also indicate that the two subunit inputs cooperate to enhance both RF center excitation and the sustained nature of ON GC response (see Fig. 10A,C).
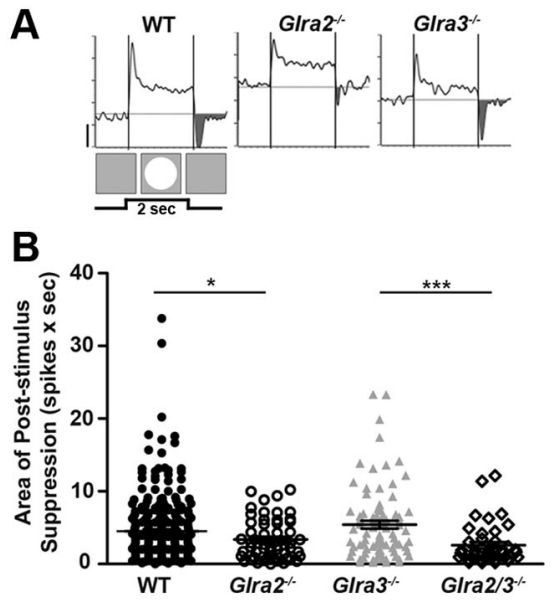

Figure 7. Only GlyR $\alpha 2$-mediated inhibition modulates poststimulus suppression in $0 \mathrm{~N} \mathrm{GCS}$. $A$, Representative PSTH responses of WT and single- and double KO ON GCs during RF center stimulation with a bright $\left(100 \mathrm{~cd} / \mathrm{m}^{2}\right)$ spot. The shaded regions represent poststimulus suppression at the offset of the stimulus (background, $20 \mathrm{~cd} / \mathrm{m}^{2}$ ). B, A scatter plot shows the distribution of the area of poststimulus suppression (spikes $\times$ sec) across genotype, which is significantly lower in Glra2 ${ }^{-1-}$ (open circles; $n=57$ ) compared to WT (closed circles; $n=284$ ) but similar in $\mathrm{Glra}^{-1-}$ ON GCs (gray triangles; $n=84$ ). The area of poststimulus suppression did not differ between and Glra2 $/ 3^{-/-}$(open diamonds; $n=41$ ) and Glra2 ${ }^{-1-}$ ON GCs, but was significantly lower compared to Glra $3^{-/-}$ON GCS. Scale bar, 20 spikes per second. ${ }^{*} p<$ $0.05 ;{ }^{* * *} p<0.001$

\section{WT ON GC response suppression at stimulus offset is specific to GlyR $\alpha 2$ inputs}

Like most mammalian GCs, the spiking activity of WT mouse ON GCs is suppressed below spontaneous levels at stimulus offset (poststimulus suppression; Fig. 7A, left). In our WT sample, ON GCs remain suppressed on average for $0.66 \mathrm{~s}$ after stimulus offset. The etiology of this suppression is not completely understood. A feedforward glycinergic input to ON GCs at stimulus offset has been shown to be generated by the Off pathway and hypothesized to use a crossover inhibitory mechanism (Chen et al., 2010).

To test the hypothesis that GlyR $\alpha 2$ and/or GlyR $\alpha 3$ mediate this input, we compared total poststimulus suppression in $\mathrm{ON}$ GCs in each single KO to WT. Consistent with a mechanism mediated by GlyR $\alpha 2$, Glra2 ${ }^{-/-}$ON GCs show less poststimulus suppression compared to WT ON GCs (Fig. 7B; $3.4 \pm 0.4$ spikes $\times$ sec, $n=57$ vs $4.5 \pm 0.3$ spikes $\times$ sec, $n=284 ; p=0.01)$. Poststimulus suppression in Glra3 ${ }^{-/-}$ON GCs was similar to that in WT (Fig. $7 B ; 5.4 \pm 0.5$ spikes $\times \sec ; n=84 ; p=0.13$ ). Poststimulus suppression in double KO ON GCs was similar to that in Glra2 ${ }^{-/-}$ON GCs (Fig. $7 B ; 2.6 \pm 0.4$ spikes $\times$ sec; $n=41$; $p=0.17)$ and significantly lower than that in Glra3 $^{-1-}$ ON GCs $(p<0.001)$. These changes most likely do not result from differences in SA levels. Although the SA of GlyR $\alpha 3$ ON GCs is lower compared to that of WT, their poststimulus suppression is similar to that of WT. In contrast, the SA of GlyR $\alpha 2$ ON GCs is similar to that of WT, whereas their poststimulus suppression is significantly less compared to WT.

In summary, both the RF center excitatory response at stimulus onset and response suppression at stimulus offset are lower in Glra2 $2^{-1-}$ ON GCs compared to WT. In $\mathrm{Glra3}^{-/-}$ON GCs, only the RF center excitatory response is lower. Combined with our results for double KO ON GCs, which show an even greater change in RF excitation than either single $\mathrm{KO}$, suggest that GlyR $\alpha 2$ and GlyR $\alpha 3$ inputs work independently in the On pathway, albeit to the same purpose, to enhance and extend RF center excitation. 
Ai

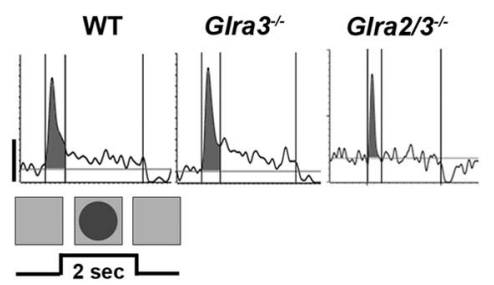

Bi
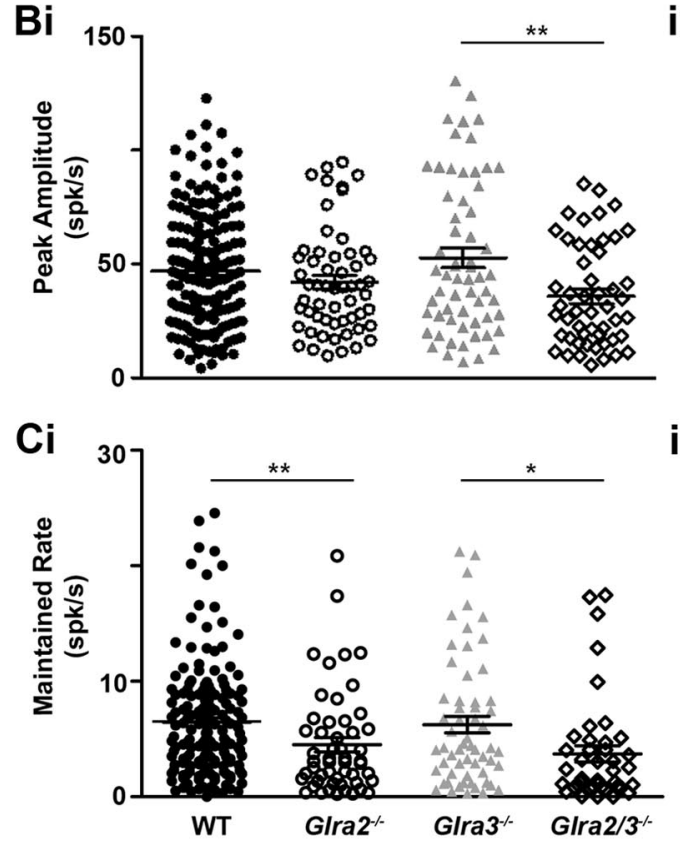

ii

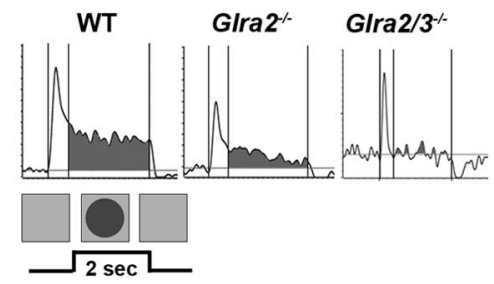

ii

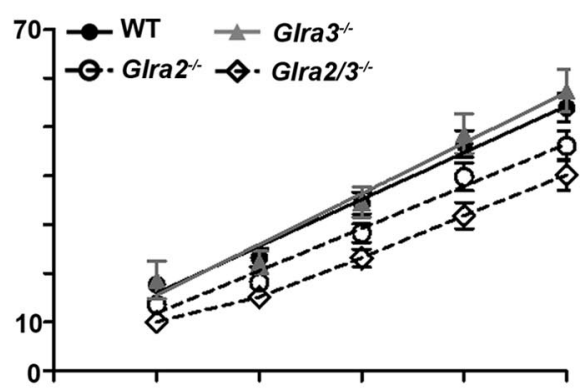

ii

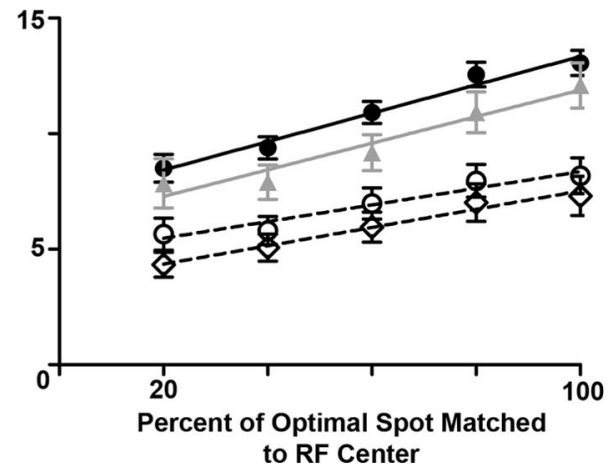

Figure 8. Only GlyR $\alpha 2$-mediated inhibition modulates the receptive field center responses of OFF GCs. Ai, Aii, Representative PSTH responses of WT and single- and double K0 0FF GCs during RF center stimulation with a dark $\left(3 \mathrm{~cd} / \mathrm{m}^{2}\right)$ spot, whose duration profile is indicated below the stimulus. The shaded regions represent the transient $(0-0.4 \mathrm{~s})$ and maintained $(0.4-2 \mathrm{~s})$ components of the excitatory response. $\boldsymbol{B i}$, The scatter plot shows the distribution of peak amplitude responses across genotype and the differences across genotype. The peak amplitudes of both $\mathrm{Glra}^{-/-}$(open

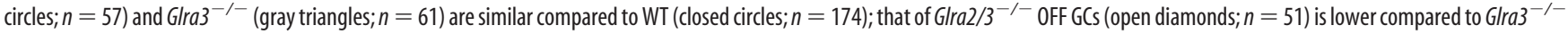
but similar compared to $\mathrm{Glra2}^{-1-}$ OFF GCS. Bii, The mean ARFs for each genotype. RF diameter is normalized so that the optimal diameter is plotted at $100 \%$. The slope represents the rate of change in peak amplitude during RF center summation and is similar between WT and single KOs $(p=0.35)$ and single and double KOs $(p=0.08)$. All curves were analyzed using a linear regression and the goodness of fit for all genotypes; $R^{2}=0.98$. Ci, The scatter plot shows the distribution of maintained firing rate across genotype and the differences across genotype. The maintained firing rates are lower in Glra ${ }^{-1-}$ OFF GCS (open circles; $n=52$ ) compared to WT (closed circles; $n=167$ ) but similar in Glra ${ }^{-1-}$ OFF GCs (gray triangles; $n=59$ ) compared to WT. The maintained firing rate

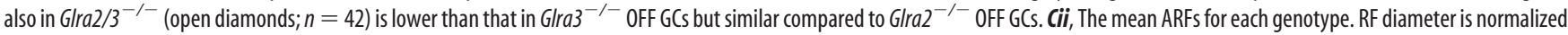
so that the optimal diameter is plotted at $100 \%$. The slope represents the rate of change in the maintained firing rate during RF center summation. The slope differs between $\mathrm{Glra2}{ }^{-/-}$and WT $0 \mathrm{FF}$ $\mathrm{GCS}(p=0.01)$ but is similar between GIra3 ${ }^{-1-}$ and WT ( $\left.p=0.71\right)$ OFF GCs. The slopes did not differ between single- and double KOs 0FF GCs $(p=0.07)$. All curves were analyzed using a linear regression and the goodness of fit for all genotypes; $R^{2}=0.95$. Scale bar, 20 spikes per second. ${ }^{*} p<0.05 ;{ }^{* *} p<0.01$.

\section{WT OFF GC temporal responses are modulated by GlyR $\alpha 2$ input}

The onset of a dark spot of standing contrast whose dimensions are within or match the RF center evokes an excitatory response in WT OFF GCs. Like their WT ON counterparts, WT OFF GCs show an initial transient peak within 0.40 s of stimulus onset (Fig. $8 A i$, left). In $77 \%$ of WT OFF GCs (134 of 174), this is followed by a maintained excitation that is temporally matched to the stimulus (Fig. 3Bi), whereas in the other $23 \%$, the excitatory response declines to SA level before stimulus offset (Fig. 3Ci). Previous publications also have described two functional types of WT OFF mouse GCs (Pang et al., 2003; Murphy and Rieke, 2006; van Wyk et al., 2009), although the stimulus durations they used were significantly shorter than $2 \mathrm{~s}$. Although the morphological identity of the GCs sampled in our extracellular optic nerve recordings are not known, we assume that they are A-type for the following reasons: Our optic nerve recordings target GCs with the largest axons (Boycott and Wässle, 1974). Optic nerve recordings only sample responses of ON- and OFF-center GCs.
Table 2. Proportion of OFF GCs with sustained or transient responses across genotype

\begin{tabular}{lllc}
\hline & Sustained (\%) & Transient (\%) & Total 0FF GCS \\
\hline WT & $77(n=134)$ & $23(n=40)$ & 174 \\
Glra2 $^{-1-*}$ & $60(n=34)$ & $40(n=23)$ & 57 \\
Glra3 $^{-/-}$ & $75(n=46)$ & $25(n=15)$ & 61 \\
Glra2/3 $^{-1-*}$ & $45(n=23)$ & $55(n=28)$ & 51 \\
\hline
\end{tabular}

The proportions of OFF sustained and OFF transient GCS are given for each genotype. The proportions of OFF sustained and OFF transient $\mathrm{GCS}$ are similar between WT and Glra ${ }^{-/-}$. In contrast, Glra2 ${ }^{-/-}$and Glra2 $/ 3^{-/}$ OFF GCs have more transient than sustained GCs compared to WT.

${ }^{*} p<0.05$.

The GCs recorded have brisk visually evoked responses and linear RF center summation (Kuffler, 1953; Enroth-Cugell and Robson, 1966).

Across Glra2 ${ }^{-/-}, \mathrm{Glra3}^{-/-}$, and Glra2/3 $3^{-/-}$OFF GCs, we found both sustained and transient GCs (Fig. 3Aii-Civ), and their proportions in $\mathrm{Glra3}^{-1-}$ OFF GCs were similar to those of WT (Table 2). In contrast, Glra2 $2^{-1-}$ and Glra2/3-1- OFF GCs had 
more transient than sustained responses compared to WT (Table 2; Glra2 $\left.{ }^{-/-}, p=0.01 ; \mathrm{Glra}_{2} / 3^{-/-}, p \leq 0.001 ; \chi^{2}\right)$. As a consequence, we did not use a sustained/transient criterion to group OFF GCs. Instead, we compared all OFF GCs in KO mice to WT to define general trends in the contribution of GlyR $\alpha 2$ and GlyR $\alpha 3$ inhibition.

\section{WT OFF GC receptive field center excitation is modulated by GlyR $\alpha 2$ input}

The transient peak response of Glra3 $^{-/-}$and WT OFF GC RF center responses at the optimal spot diameter were similar (Fig. $8 \mathrm{Ai}, \mathrm{Bi} ; 52.7 \pm 4.3 \mathrm{spikes} / \mathrm{s}, n=61$ vs $47.0 \pm 2.0$ spikes/s, $n=174$; $p=0.22$ ), as were their maintained firing rates (Fig. $8 \mathrm{Ai}, \mathrm{Ci} ; 6.2 \pm$ 0.7 spikes/s, $n=59$ vs $6.5 \pm 0.4$ spikes/s, $n=167 ; p=0.80)$. These similarities were maintained across all spot diameters within the RF center (Fig. 8 Bii,Cii; $p>0.05$ for both). No other differences were noted in any aspect of the RF center response at stimulus onset or offset, leading to the conclusion that GlyR $\alpha 3$ input does not modulate OFF GC RF center responses.

The transient peak response of Glra2 ${ }^{-1-}$ OFF GCs at all spot diameters within the RF center was similar to that of WT (Fig. $8 \mathrm{Bi}$,Bii; optimal, $42.0 \pm 3.0$ spikes $/ \mathrm{s} ; n=57 ; p=0.20$; all diameters, $p>0.05)$. The maintained firing rates of $\mathrm{Glra2}^{-/-}$OFF GC RF center excitatory responses were significantly lower than those of WT OFF GCs both at the optimal diameter (Fig. 8 Aii,Ci; $4.5 \pm$ 0.6 spikes/s, $n=52$ vs $6.5 \pm 0.4$ spikes/s, $n=167 ; p=0.009)$ and across all spot diameters within the RF center (Fig. 8Cii; $p<$ 0.001). The difference in the maintained response component causes the increase in transient GlyR $\alpha 2$ OFF GCs compared to WT described above.

Both the peak RF center response of double KO OFF GCs (Fig. $8 \mathrm{Ai}, \mathrm{Bi}$; peak, $36.0 \pm 3.1 \mathrm{spikes} / \mathrm{s} ; n=51 ; p=0.17)$ and the maintained response were similar to those of $\mathrm{Glra2}^{-/-}$OFF GCs (Fig. 8Ci; maintained, $3.7 \pm 0.7$ spikes $/ \mathrm{s} ; n=42 ; p=0.40$ ). Consequently, they were significantly lower compared to Glra3 $^{-/-}$OFF GCs ( $p=0.002$ and $p=0.02$, respectively).

OFF GCs also exhibit response suppression at stimulus offset (Fig. 9A). Glra2 ${ }^{-1-}$ OFF GCs had significantly less poststimulus suppression compared to WT (Fig. 9B; $0.8 \pm 0.1$ spikes $\times$ sec, $n=$ 56 vs $1.7 \pm 0.1$ spikes $\times$ sec, $n=168 ; p<0.001)$. Poststimulus suppression in Glra3 ${ }^{-1-}$ OFF GCs was similar to that in WT OFF GCs (Fig. 9B; $2.2 \pm 0.3$ spikes $\times$ sec; $n=59 ; p=0.06$ ). Poststimulus suppression in double KO OFF GCs $(1.1 \pm 0.2$ spikes $\times \sec ; n=50)$ was similar to that in $\mathrm{Glra}^{-/-}$OFF GCs $(p=0.10)$ and, as a consequence, lower than in $\mathrm{Glra3}^{-/-} \mathrm{OFF}$ GCs $(p=0.001)$.

Our results demonstrate a novel role for GlyR $\alpha 2$-mediated inhibition in modulating the RF center response of WT OFF GCs and relative to GlyR $\alpha 3$-mediated inhibition, the only one that modulates WT OFF GC responses. The exact role of GlyR $\alpha 1$ input, which is the predominant glycinergic synaptic input to mouse rod and cone Off bipolar cells as well as A-type GCs (Majumdar et al., 2007) remains to be defined and will require conditional elimination of its expression. The differences that we observed for GlyR $\alpha 2$ in ON GCs, namely, less excitation and less suppression, are the same as those we observe in OFF GCs. This indicates that GlyR $\alpha 2$ uses a similar inhibitory mechanism in both pathways to the same end, to enhance the excitatory RF center response and match it to stimulus duration.
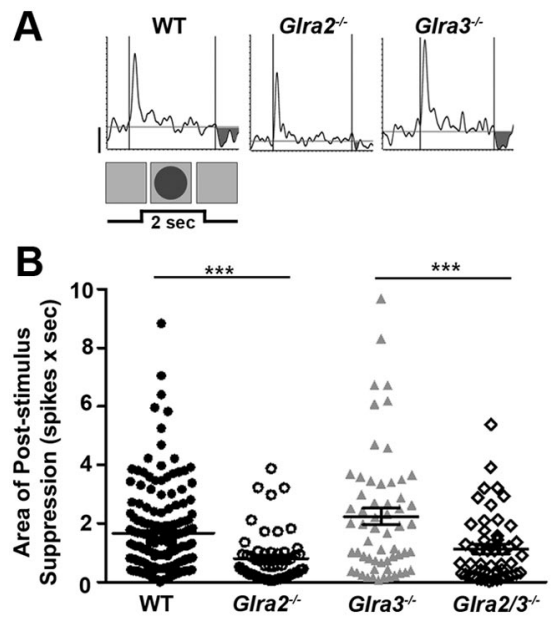

Figure 9. Only GlyR $\alpha 2$-mediated inhibition modulates poststimulus suppression in OFF GCs. $A$, Representative PSTH responses of WT and single KO OFF GCs during receptive field center stimulation with a dark $\left(3 \mathrm{~cd} / \mathrm{m}^{2}\right)$ spot. The shaded regions represent poststimulus suppression at the offset of the stimulus (background, $20 \mathrm{~cd} / \mathrm{m}^{2}$ ). B, A scatter plot shows the distribution of the area of poststimulus suppression (spikes $\times \mathrm{sec}$ ) across genotype, which is lower in Glra2 ${ }^{-/-}$(open circles; $n=56$ ) compared to WT OFF GCs (closed circles; $n=168$ ) but similar in Glra3 $^{-1-}$ OFF GCs (gray triangles; $n=59$ ). The area of poststimulus suppression in Glra2/ $3^{-1-}$ OFF GCs (open diamonds; $n=50$ ) is lower than in $\mathrm{Glra}^{-1-}$ OFF GCs but similar to that in Glra2 $2^{-/-}$OFF GCs. Scale bar, 20 spikes per second. ${ }^{* *} p<0.001$.

\section{Discussion}

GlyR $\alpha 2$ and GlyR $\alpha 3$ subunit-specific modulation of $\mathrm{ON}$ and OFF GC RF center temporal responses

Our results show GlyR $\alpha 2$ subunit-specific control over the maintained component of the RF center response of OFF GCs to stimulus onset and poststimulus suppression. In this pathway, GlyR $\alpha 2$ functions to enhance GC RF center excitation and temporally match the excitatory response to stimulus duration. In ON GCs, temporal response components are governed by both GlyR $\alpha 2$ and GlyR $\alpha 3$ subunit inputs, which appear to contribute separately but function together to perform the same response enhancement and temporal matching. We found no evidence of either subunit input alone mediating spatial aspects of the RF response, which is consistent with an established role of glycinergic inhibition in the modulation of temporal and not spatial properties of the GC visual response (Bolz et al., 1985; Stone and Pinto, 1992; O’Brien et al., 2003; Chen et al., 2010).

\section{GlyR $\alpha 2$ and GlyR $\alpha 3$ subunits modulate inhibition slower than receptor kinetics}

In Glra2 $2^{-/-}$OFF GCs, a lower maintained excitatory response compared to WT results in more transient responses both in Glra2 ${ }^{-/-}$and Glra2/3 $3^{-/-}$double KO mice. Spontaneous GlyR $\alpha 1$ and GlyR $\alpha 3$ IPSCs (Ivanova et al., 2006; Majumdar et al., 2007; Weiss et al., 2008) and light-evoked glycinergic IPSCs (Pang et al., 2007; Manookin et al., 2008) have been shown in cellular components of the Off pathway. Thus, our results define a new role for GlyR $\alpha 2$ inhibition in shaping the response of WT OFF GCs to match stimulus duration.

GlyR $\alpha 2$ and GlyR $\alpha 3$ control of the maintained response is slow (between 0.4 and $2 \mathrm{~s}$ ) compared to their receptor kinetics (mean decay $\tau$, GlyR $\alpha 2, \sim 27 \mathrm{~ms}$; GlyR $\alpha 3, \sim 11 \mathrm{~ms}$ ) (Weiss et al., 2008). This difference is most easily explained by a prolonged or asynchronous release of presynaptic neurotransmitter that shapes the inhibitory input controlling the maintained response, similar to the timing of inhibitory inputs that shape BC light- 


\section{A GlyRa2 Crossover Inhibition Off to On pathway}

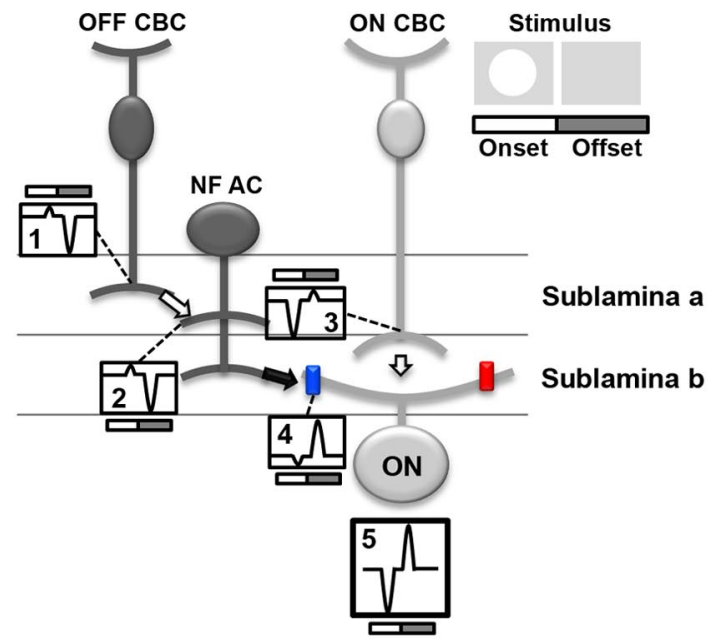

\section{B GlyRa2 Crossover Inhibition On to Off pathway}

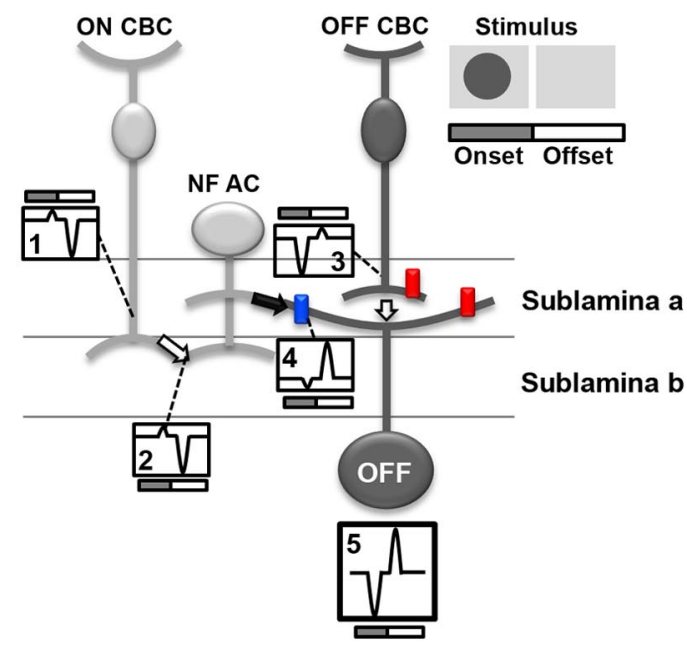

\section{GlyRa3 Serial Inhibition in the On pathway}

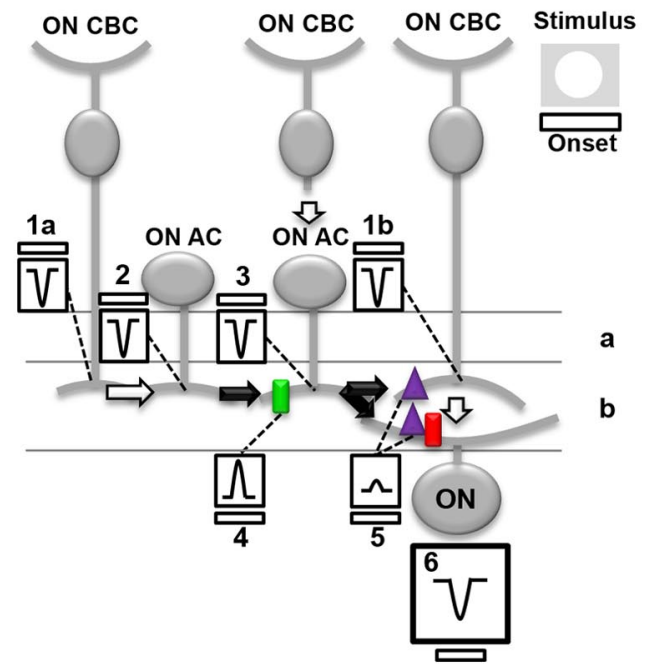

evoked inhibition (Tachibana, 1999; Eggers and Lukasiewicz, 2006; Eggers et al., 2007).

GlyR $\alpha 2$ and GlyR $\alpha 3$ enhance $\mathrm{ON}$ and OFF GC RF center excitation through separate inhibitory mechanisms GlyR $\alpha 2$ mediates crossover inhibition in both the On and Off pathways

The absence of GlyR $\alpha 2$ expression creates the same changes in ON and OFF GC RF center responses. Both the transient and sustained components of the RF center excitatory responses at stimulus onset and poststimulus suppression are significantly lower than those of WT. Less excitation is inconsistent with the removal of direct inhibition. Removal of serial inhibitory input will result in less excitation at stimulus onset; however poststimulus suppression, arising from inhibitory inputs in the opposite pathway, should be unchanged. The most parsimonious explanation for our result is that GlyR $\alpha 2$ meditates crossover inhibition from the Off to the On as well as the On to Off WT pathways. Glycinergic crossover inhibition has

\section{$\leftarrow$}

currents, as described in Results. $\boldsymbol{A}$, GlyR $\alpha 2$ mediates crossover inhibition from the Off to the On pathway. At stimulus onset (white bar), the NF AC is driven by an OFF CBC (Box 1), which produces a small outward current (Box 2). The NF AC, using a crossover inhibitory mechanism, inverts the polarity of the current via Gly $R \alpha 2$ (blue), producing a current in the ON GC (Box 4) that enhances the current from its presynaptic ON CBC (Box 3). At stimulus offset (gray bar), a similar mechanism transfers an inverted polarity current from the off to the 0 n pathway, which enhances poststimulus suppression. The spiking response of the $\mathrm{GC}$ is governed by the summation of the inputs (Box 5). B, GlyR $\alpha 2$ mediates crossover inhibition from the 0 n to the Off pathway. Similar to the description in $A$, at stimulus onset (gray bar), the NF $A C$ is driven by an $\mathrm{ON} C B C$ (Box 1), which produces a small outward current (Box 2). The NF AC, using a crossover inhibitory mechanism inverts the polarity of the current via GlyR $\alpha 2$ (blue), producing a current in the OFF GC (Box 4) that enhances the current from its presynaptic OFF CBC (Box 3). At stimulus offset (white bar), a similar mechanism transfers an inverted polarity current from the off to the 0 n pathway, which enhances poststimulus suppression. The spiking response of the $\mathrm{GC}$ is governed by the summation of the inputs (Box 5). C, GlyR $\alpha 3$ mediates serial inhibition in the On pathway. At stimulus onset (white bar), ON CBCs depolarize (Boxes $1 \mathrm{a}$ and $1 \mathrm{~b}$ represent currents in all $\mathrm{CBCS}$ ). The $\mathrm{ON}$ CBC (far left) targets an $\mathrm{ON} A C$ producing an inward current (Box 2). Using GlyR $\alpha 3$ (green), this distal ON AC produces an outward current in the proximal ON AC (Box 4), which is summed with the inward current produced via input from its presynaptic ON CBC (Box 3). The proximal ON AC targets either the $\mathrm{ON} \mathrm{CBC} \mathrm{(far} \mathrm{right)} \mathrm{at} \mathrm{a} \mathrm{GABA} \mathrm{receptor} \mathrm{(purple)} \mathrm{or} \mathrm{an} \mathrm{ON} \mathrm{GC}$ at either a GABA receptor (purple) or a GlyR $\alpha 1 R$ (red). Because the proximal ON AC output is controlled by its ON AC inhibitory input, the outward current (Box 5) is small. Depending on the postsynaptic target of the proximal ON AC, the GCs total current (Box 6) reflects either the input from its presynaptic $C B C$ (Box $1 \mathrm{~b})$ or from the inputs from its presynaptic $C B C$ and $O N A C$ (Box 5).
Figure 10. GlyR $\alpha 2$ and GlyR $\alpha 3$ mediate inhibition using different inhibitory circuits. $\mathbf{A}-\boldsymbol{C}$, Schematic diagrams of the proposed roles of GlyR $\alpha 2$ and GlyR $\alpha 3$ in the WT mouse 0 n and Off retinal pathways are shown. Each diagram illustrates a basic retina circuit that is consistent with the results presented. The diagrams in the top right corners illustrate the stimulus and the timing of its onse and offset. Within each circuit, the numbered boxes represent the current evoked at stimulus onset and offset. Nota bene: Currents are represented with transient time course for simplicity. The currents in the boxes do not reflect the temporal properties of the 
been shown to augment both excitation at stimulus onset and suppression at stimulus offset in rabbit GCs (Roska and Werblin, 2001; Roska et al., 2006; Molnar and Werblin, 2007; Hsueh et al., 2008; Molnar et al., 2009; Chen et al., 2010). In addition, mouse OFF GCs receive glycinergic inhibitory inputs from the On pathway (van Wyk et al., 2009).

Figure $10 \mathrm{~A}$ illustrates a circuit in which GlyR $\alpha 2$ mediates crossover input from the Off to On pathway. The GlyR $\alpha 2$ receptor is positioned as the target of a narrow field (NF) AC, where it mediates inhibition to an ON GC. We have eliminated ON cone BCs (CBCs) as the target because they lack spontaneous and evoked glycinergic IPSCs (Ivanova et al., 2006; Eggers et al., 2007). At the onset of a bright-spot stimulus (currents under white portion of stimulus profile bars), the primary inward current that governs excitation in ON GCs (Fig. 10A, Box 5) arises from $\mathrm{ON}$ CBCs (Box 3). In the Off pathway, stimulus onset produces a small outward current in OFF CBCs (Box 1) that provides input and produces a response in the NF AC (Box 2). Crossover inhibition inverts this current, and the NF AC synaptic input produces a current in the ON GC (Box 4) with the same sign as its ON CBC input (Box 3). In WT ON GCs, this mechanism enhances excitation, and in the absence of GlyR $\alpha 2$ input, only the ON CBC input drives the ON GC response, and the stimulus evokes less excitation. The offset of the bright spot (currents under the gray portion of the stimulus profile bars) eliminates the excitatory input from the ON CBC and induces a small outward current (Box 3). At the same time, OFF CBC excitation elicits a large inward current in the NF AC (Box 2). Crossover inhibition inverts current polarity and the NF AC input produces a current in the ON GC (Box 4) of the same sign as its ON CBC input (Box 3). This enhances response suppression in WT ON GCs (Box 5), and the absence of GlyR $\alpha 2$ input reduces suppression.

Changes in RF center responses of OFF GCs in the absence of GlyR $\alpha 2$ are the same as those in ON GCs, which suggests a similar GlyR $\alpha 2$ inhibitory mechanism in the On and Off pathways (Fig. $10 \mathrm{~B}$ ). Although the receptor subunit was unknown at the time, On-to-Off pathway inhibition was predicted to occur via an intermediate glycinergic AC in cat GCs (Wässle et al., 1986). Given its localization throughout the IPL (Heinze et al., 2007), our results suggest that GlyR $\alpha 2$ is a subunit that mediates inhibitory inputs from the On to the Off pathway and vice versa.

Figure $10 B$ illustrates GlyR $\alpha 2$-mediated crossover input from the On to the Off pathway. Here the GlyR $\alpha 2$ receptor is the target of a NF AC for two reasons. All spontaneous and glycine evoked IPSCs in OFF CBCs are mediated by GlyR $\alpha 1$ (Ivanova et al., 2006). Glycinergic IPSCs with slower kinetics remain in A2 OFF GCs in the absence of their dominant GlyR $\alpha 1$ synaptic input (Majumdar et al., 2007). The onset of a dark spot initiates excitation in OFF CBCs (currents under gray portion of stimulus profile bars). Using a NF AC, the same crossover inhibitory mechanisms described for the Off to the On pathway (Fig. 10A) enhance the RF excitatory response as well as response suppression in WT OFF GCs. Again, the absence of GlyR $\alpha 2$ input lowers response excitation and suppression. It should be noted that concatenated circuits have been observed previously (Anderson et al., 2011). If they provide input to the NF AC that mediates crossover inhibition, then the position of the GlyR $\alpha 2$ receptor could differ from our placement in Figure 10, $A$ and $B$. What is not in question is that our observations are consistent with a role of the GlyR $\alpha 2$ receptor and a crossover inhibitory circuit that contributes to GC RF center response enhancement.
GlyR $\alpha 3$ mediates serial inhibition in the On pathway

Both RF center transient and maintained response components of $\mathrm{Glra3}^{-/-}$ON GCs are lower than those of WT. The absence of GlyR $\alpha 3$ inhibition does not affect poststimulus suppression, and we therefore predict that GlyR $\alpha 3$ uses a different mechanism, serial inhibition, in the On pathway. GABAergic feedback to the RF center of rabbit local edge detector GCs is controlled by serial glycinergic inhibition (Russell and Werblin, 2010), and this mechanism also governs salamander GC responses (Zhang et al., 1997).

Figure $10 C$ illustrates GlyR $\alpha 3$-mediated serial inhibitory input within the On pathway. This pathway is shown separately for clarity, although GlyR $\alpha 2$ and GlyR $\alpha 3$ could work together in the same circuit. The GlyR $\alpha 3$ receptor is located on the ON AC most proximal to the ON GC, as the target of a distal ON AC. All AC processes are confined to the On sublaminae because Glra3 $^{-1-}$ OFF GC RF responses are similar to those of WT. ON CBCs only show GABAergic sIPSCs; therefore, if they are the target, then the proximal ON AC is GABAergic (Eggers et al., 2007), whereas if the target is an ON GC, the proximal ON AC can be glycinergic targeting GlyR $\alpha 1$ Rs (Ivanova et al., 2006) or GABAergic targeting $\mathrm{GABA}_{\mathrm{A}}$ Rs (Eggers et al., 2007). At the onset of a bright-spot stimulus (Fig. 10C, currents under white stimulus profile bars), an excitatory response arises in ON CBCs (Boxes 1a, 1b), resulting in inward currents in ON ACs (Boxes 2, 3). GlyR $\alpha 3$-mediated inhibition from the distal ON AC (Box 4) reduces the inward current in the proximal ON AC (Box 5). This reduces its inhibitory output to the postsynaptic target (WT ON CBC or GC). The absence of GlyR $\alpha 3$ expression eliminates the inhibitory input to the proximal $\mathrm{AC}$ and increases its inhibitory input. When its target is the $\mathrm{ON} \mathrm{CBC}$, this reduces excitatory input to the ON GC. When its target is the ON GC, excitation decreases because direct inhibition increases. Regardless, the absence of GlyR $\alpha 3$ expression reduces stimulus evoked spiking in the ON GC (Box 6).

\section{Summary}

Our findings extend the previous literature that demonstrated a significant role of glycinergic inhibition in shaping visual responses in the retina. Our results define novel roles of two glycine subunits not only in their selectivity within the On and Off pathways, but also in the specific inhibitory mechanisms that they employ to modulate GC visual function. One question arises whenever the role of ACs in retinal function is discussed: Why are there so many diverse AC classes? In light of this question, our results could be interpreted as evidence to support the idea that multiple ACs are required to target each subunit-specific input within the retinal parallel pathways and that different ACs are required to modulate both the transient and sustained aspects of the visual response.

\section{References}

Anderson JR, Jones BW, Watt CB, Shaw MV, Yang JH, Demill D, Lauritzen JS, Lin Y, Rapp KD, Mastronarde D, Koshevoy P, Grimm B, Tasdizen T, Whitaker R, Marc RE (2011) Exploring the retinal connectome. Mol Vis 17:355-379.

Barthel LK, Raymond PA (1990) Improved method for obtaining 3-microns cryosections for immunocytochemistry. J Histochem Cytochem 38:1383-1388.

Bolz J, Thier P, Voigt T, Wässle H (1985) Action and localization of glycine and taurine in the cat retina. J Physiol 362:395-413.

Bormann J (2000) The "ABC" of GABA receptors. Trends Pharmacol Sci $21: 16-19$.

Boycott BB, Wässle H (1974) The morphological types of ganglion cells of the domestic cat's retina. J Physiol 240:397-419.

Caldwell JH, Daw NW (1978) Effects of picrotoxin and strychnine on rabbit 
retinal ganglion cells: changes in centre surround receptive fields. J Physiol 276:299-310.

Chen X, Hsueh HA, Greenberg K, Werblin FS (2010) Three forms of spatial temporal feedforward inhibition are common to different ganglion cell types in rabbit retina. J Neurophysiol 103:2618-2632.

Chen X, Hsueh HA, Werblin FS (2011) Amacrine-to-amacrine cell inhibition: Spatiotemporal properties of GABA and glycine pathways. Vis Neurosci 28:193-204.

Cook PB, McReynolds JS (1998) Modulation of sustained and transient lateral inhibitory mechanisms in the mudpuppy retina during light adaptation. J Neurophysiol 79:197-204.

Cui J, Ma Y-P, Lipton SA, Pan Z-H (2003) Glycine receptors and glycinergic synaptic input at the axon terminals of mammalian retinal rod bipolar cells. J Physiol 533 3:895-909.

Eggers ED, Lukasiewicz PD (2006) Receptor and transmitter release properties set the time course of retinal inhibition. J Neurosci 26:9413-9425.

Eggers ED, Lukasiewicz PD (2010) Interneuron circuits tune inhibition in retinal bipolar cells. J Neurophysiol 103:25-37.

Eggers ED, Lukasiewicz PD (2011) Multiple pathways of inhibition shape bipolar cell responses in the retina. Vis Neurosci 28:95-108.

Eggers ED, McCall MA, Lukasiewicz PD (2007) Presynaptic inhibition differentially shapes transmission in distinct circuits in the mouse retina. J Physiol 582:569-582.

Enroth-Cugell C, Robson JG (1966) The contrast sensitivity of retinal ganglion cells of the cat. J Physiol 187:517-552.

Enz R, Bormann J (1995) Expression of glycine receptor subunits and gephyrin in single bipolar cells of the rat retina. Vis Neurosci 12:507-517.

Flores-Herr N, Protti DA, Wässle H (2001) Synaptic currents generating the inhibitory surround of ganglion cells in the mammalian retina. J Neurosci 21:4852-4863.

Gregg RG, Kamermans M, Klooster J, Lukasiewicz PD, Peachey NS, Vessey KA, McCall MA (2007) Nyctalopin expression in retinal bipolar cells restores visual function in a mouse model of complete $\mathrm{X}$-linked congenital stationary night blindness. J Neurophysiol 98:3023-3033.

Grimes WN, Zhang J, Graydon CW, Kachar B, Diamond JS (2010) Retinal parallel processors: more than 100 independent microcircuits operate within a single interneuron. Neuron 65:873-885.

Grudzinska J, Schemm R, Haeger S, Nicke A, Schmalzing G, Betz H, Laube B (2005) The beta subunit determines the ligand binding properties of synaptic glycine receptors. Neuron 45:727-739.

Harvey RJ, Depner UB, Wässle H, Ahmadi S, Heindl C, Reinold H, Smart TG, Harvey K, Schütz B, Abo-Salem OM, Zimmer A, Poisbeau P, Welzl H, Wolfer DP, Betz H, Zeilhofer HU, Müller U (2004) GlyR alpha3: an essential target for spinal PGE2-mediated inflammatory pain sensitization. Science 304:884-887.

Haverkamp S, Müller U, Harvey K, Harvey RJ, Betz H, Wässle H (2003) Diversity of glycine receptors in the mouse retina: localization of the alpha3 subunit. J Comp Neurol 465:524-539.

Heinze L, Harvey RJ, Haverkamp S, Wässle H (2007) Diversity of glycine receptors in the mouse retina: localization of the alpha4 subunit. J Comp Neurol 500:693-707.

Hsueh HA, Molnar A, Werblin FS (2008) Amacrine-to-amacrine cell inhibition in the rabbit retina. J Neurophysiol 100:2077-2088.

Ivanova E, Müller U, Wässle H (2006) Characterization of the glycinergic input to bipolar cells of the mouse retina. Eur J Neurosci 23:350-364.

Kuffler SW (1953) Discharge patterns and functional organization of mammalian retina. J Neurophysiol 16:37-68.

MacNeil MA, Masland RH (1998) Extreme diversity among amacrine cells: implications for function. Neuron 20:971-982.

MacNeil MA, Heussy JK, Dacheux RF, Raviola E, Masland RH (1999) The shapes and numbers of amacrine cells: matching of photofilled with Golgi-stained cells in the rabbit retina and comparison with other mammalian species. J Comp Neurol 413:305-326.
Majumdar S, Heinze L, Haverkamp S, Ivanova E, Wässle H (2007) Glycine receptors of A-type ganglion cells of the mouse retina. Vis Neurosci 24:471-487.

Manookin MB, Beaudoin DL, Ernst ZR, Flagel LJ, Demb JB (2008) Disinhibition combines with excitation to extend the operating range of the OFF visual pathway in daylight. J Neurosci 28:4136-4150.

Menger N, Pow DV, Wässle H (1998) Glycinergic amacrine cells of the rat retina. J Comp Neurol 401:34-46.

Molnar A, Werblin F (2007) Inhibitory feedback shapes bipolar cell responses in the rabbit retina. J Neurophysiol 98:3423-3435.

Molnar A, Hsueh HA, Roska B, Werblin FS (2009) Crossover inhibition in the retina: circuitry that compensates for nonlinear rectifying synaptic transmission. J Comput Neurosci 27:569-590.

Murphy GJ, Rieke F (2006) Network variability limits stimulus-evoked spike timing precision in retinal ganglion cells. Neuron 52:511-524.

O'Brien BJ, Richardson RC, Berson DM (2003) Inhibitory network properties shaping the light evoked responses of cat alpha retinal ganglion cells. Vis Neurosci 20:351-361.

Pang JJ, Gao F, Wu SM (2003) Light-evoked excitatory and inhibitory synaptic inputs to ON and OFF alpha ganglion cells in the mouse retina. J Neurosci 23:6063-6073.

Pang JJ, Abd-El-Barr MM, Gao F, Bramblett DE, Paul DL, Wu SM (2007) Relative contributions of rod and cone bipolar cell inputs to AII amacrine cell light responses in the mouse retina. J Physiol 580:397-410.

Roska B, Werblin F (2001) Vertical interactions across ten parallel, stacked representations in the mammalian retina. Nature 410:583-587.

Roska B, Nemeth E, Orzo L, Werblin FS (2000) Three levels of lateral inhibition: a space-time study of the retina of the tiger salamander. J Neurosci 20:1941-1951.

Roska B, Molnar A, Werblin FS (2006) Parallel processing in retinal ganglion cells: how integration of space-time patterns of excitation and inhibition form the spiking output. J Neurophysiol 95:3810-3822.

Russell TL, Werblin FS (2010) Retinal synaptic pathways underlying the response of the rabbit local edge detector. J Neurophysiol 103:2757-2769.

Sagdullaev BT, McCall MA (2005) Stimulus size and intensity alter fundamental receptive-field properties of mouse retinal ganglion cells in vivo. Vis Neurosci 22:649-659.

Sagdullaev BT, DeMarco PJ, McCall MA (2004) Improved contact lens electrode for corneal ERG recordings in mice. Doc Ophthalmol 108:181-184.

Sassoè-Pognetto M, Wässle H, Grünert U (1994) Glycinergic synapses in the rod pathway of the rat retina: cone bipolar cells express the $\alpha 1$ subunit of the glycine receptor. J Neurosci 14:5131-5146.

Stone C, Pinto LH (1992) Receptive field organization of retinal ganglion cells in the spastic mutant mouse. J Physiol 456:125-142.

Tachibana M (1999) Regulation of transmitter release from retinal bipolar cells Prog Biophys Mol Biol 72:109-133.

van Wyk M, Wässle H, Taylor WR (2009) Receptive field properties of ONand OFF-ganglion cells in the mouse retina. Vis Neurosci 26:297-308.

Wässle H, Schäfer-Trenkler I, Voigt, T (1986) Analysis of a glycinergic inhibitory pathway in the cat retina. J Neurosci 6:594-604.

Wässle H, Heinze L, Ivanova E, Majumdar S, Weiss J, Harvey RJ, Haverkamp $S$ (2009) Glycinergic transmission in the mammalian retina. Front Mol Neurosci 2:1-12.

Webb TI, Lynch JW (2007) Molecular pharmacology of the glycine receptor chloride channel. Curr Pharm Des 13:2350-2367.

Weiss J, O'Sullivan GA, Heinze L, Chen HX, Betz H, Wässle H (2008) Glycinergic input of small-field amacrine cells in the retinas of wildtype and glycine receptor deficient mice. Mol Cell Neurosci 37:40-55.

Young-Pearse TL, Ivic L, Kriegstein AR, Cepko CL (2006) Characterization of mice with targeted deletion of glycine receptor alpha 2. Mol Cell Biol 26:5728-5734.

Zhang J, Jung CS, Slaughter MM (1997) Serial inhibitory synapses in retina. Vis Neurosci 14:553-563. 